\title{
Stationary Analysis of Fluid Level Dependent Bounded Fluid Models*
}

\author{
M. Gribaudo ${ }^{[1]}$, M. Telek ${ }^{[2]}$ \\ [1] Dipartimento di Informatica, Università di Torino, Torino, Italy \\ [2] Department of Telecommunications, Technical University of Budapest, \\ Budapest, Hungary \\ e-mail: marcog@di.unito.it, telek@hit.bme.hu
}

\begin{abstract}
In stochastic fluid models the drift at which the fluid level changes in the fluid buffer and the generator of the underlying process might depend on the discrete state of the system and on the fluid level itself. In this paper we analyze the stationary behaviour of finite buffer Markov fluid models in which the drift and the generator of the underlying continuous time Markov chain (CTMC) depends on both of these parameters. Especially, the case when the drift changes sign at a given fluid level is considered. This case requires a particular treatment, because at this fluid level probability mass might develop. When dealing with sign changes, new problems that were not addressed in previous works arises in the solution process. The set of stationary equations is provided and a transformation of the unknowns is applied to obtain a solvable system description. Numerical examples introduce the behaviour of fluid systems with various discontinuities and sign changes of the drift.
\end{abstract}

Key words: Stochastic fluid model, stationary distribution.

\section{Introduction}

There is a wide range of performance analysis problems in which the system behaviour is Markovian over a mixed discrete and continuous state space

*This work is partially supported by the Italian-Hungarian bilateral R\&D program and by OTKA grant n. T-34972. 
and there are several stochastic models developed for the analysis of these systems. Examples of such models are Markov reward models, Markov modulated Brownian motion and stochastic fluid models. The case when the variation of the continuous system parameter is bounded (at least from one side) is commonly referred to as fluid model.

The complexity of fluid models depends on several model features among which one of the most important is the dependency structure of the discrete and continuous part of the model. In case of several real life examples the discrete state process of the system form a continuous time Markov chain (CTMC) on its own (independent of the continuous part) and the continuous system variable (commonly referred to as fluid level) evolves according to the discrete system state [10]. In these cases the marginal distribution of the discrete system state can be analyzed independent of the fluid part of the model. In this paper we consider the more complex case when the discrete and the continuous part of the model are mutually dependent, such that none of them can be analyzed independently [7, 3].

Further classifying model feature is the kind of dependence of the fluid increment on the discrete and the continuous part of the system. In first order fluid models there is a deterministic relation between the discrete state and the fluid increment $[1,10]$, while in second order fluid models this relation is stochastic (the discrete state determines the mean and the variance of the fluid increment) $[3,12]$.

The analytical description of fluid models is provided by a set of partial differential equations (PDEs) and associated boundary conditions. For nontrivial cases the symbolic solution of the system equations is not available. Instead, the transient analysis is carried out with numerical solution of the PDEs considering the boundary conditions and the initial distribution of the system $[7,12]$. The availability of an initial distribution, to start the numerical solution, is a significant advantage of the transient analysis of fluid models compared with their stationary analysis. In case of stationary analysis we can eliminate the derivative of the time variable, which simplifies the PDEs to ordinary differential equations (ODEs), but there is no initial condition available for the numerical solution of the ODEs. It is a significant drawback of the stationary analysis of fluid models. To obtain the "initial conditions" of the ODEs a set of equations is composed based on the boundary equations, the solution of the ODE and a normalizing condition. For example, in case of fluid level independent transition matrix and fluid drift vector, the solution of the ODE is assumed to be exponential and the coefficients of the exponential solution are calculated from the boundary equations and the normalizing condition [10]. Unfortunately, this effective solution approach is not applicable when the transition matrix or the fluid drift vector depends 
on the fluid level.

One of the most important result of this paper is that the nature of the considered problem inhibits the use of previously applied model description with fluid density and mass probability and it requires the introduction of new measures to analyze the system. Conventional techniques, like the one proposed in [6], applied in this context, produce systems of equations that cannot be solved.

The rest of the paper is organized as follows. Section 2 compare the results proposed in this paper with the one previously presented in the literature. Section 3 introduces the considered fluid model and its transient description over the continuous regions. Section 4 provides the boundary equations to describe the behaviour at discontinuities and boundaries. The number of obtained equations and unknowns are considered in section 5. A modified set of equations is introduced in section 6 and the properties of the obtained set of equations are provided in 7 . Section 8 discusses the case when there are states with zero fluid rate and section 9 provides the normalizing conditions. A set of numerical examples demonstrate the applicability of the proposed method in section 10 and the paper is concluded in section 11 .

\section{Related works}

There are several papers dealing with the transient analysis of fluid level dependent transition matrix and fluid drift vector $[12,7,3]$, but rather few results available for the stationary analysis of these systems mainly due to the mentioned lack of an initial condition. The approach to compose the set of equation characterizing the initial condition without using the assumption on the exponential solution of the set of ODEs was provided in [6], but the first real non-exponential ODE system was introduced in [5], because [6] still assumed fluid level independent transition matrix and fluid drift vector.

There is a meaningful restriction applied in [5]. The elements of the fluid drift vector do not change sign. This restriction results that the fluid level distribution is continuous (i.e., there is no probability mass of the fluid distribution) between the fluid bounds.

The transient behaviour of the case when the fluid drift changes sign in a particular state was studied in [3]. In this paper we use slightly different assumptions (not the discontinuity, but the sign change of the fluid drift function separates "continuous pieces" and the behaviour of the "isolating state" is different in this work) and provide the stationary analysis of first order fluid models with mutually dependent continuous and discrete parts.

An analytical solution to fluid models with state dependent flow rate has 
been proposed in [8]. In that case, the system was analyzed both in transient and steady state, using Laplace Transforms. With respect to that work, we restrict our analysis to steady state, but we allow the background process to depend on the fluid level, and consider sign changes. Fluid model with fluid dependent rates, were also addressed in [9]. In that case, a system with two fluid variables, with interdependent fluid rates where considered. Our work will focus only on systems with a single fluid variable, but will allow them to be driven by a more complex stochastic process. The problem of dealing with states with zero flow rate was also considered in [2] and in [11]. Here we will use a similar solution to remove zero flow rate states from a model.

\section{$3 \quad$ Stationary description of fluid models with single finite fluid buffer}

The $Z(t)=\{M(t), X(t) ; t \geq 0\}$ process represents the state of a fluid model with single fluid buffer, where $M(t) \in \mathcal{S}$ is the (discrete) state of the environment process and $X(t) \in[0, B]$ is the fluid level of the fluid buffer at time $t$. $\mathcal{S}$ denotes the finite set of states of the environment and $B$ the maximum fluid level. The fluid level distribution might have probability masses at particular fluid levels and it is continuous between these levels. We define $\hat{\pi}_{j}(t, x)$ and $\hat{c}_{j}(t, x)$ to describe the continuous part and the probability masses of the transient fluid distribution as follows

$$
\begin{gathered}
\hat{\pi}_{j}(t, x)=\lim _{\Delta \rightarrow 0} \frac{\operatorname{Pr}(M(t)=j, x \leq X(t)<x+\Delta)}{\Delta}, \\
\hat{c}_{j}(t, x)=\operatorname{Pr}(M(t)=j, X(t)=x),
\end{gathered}
$$

and, assuming the system converges to a single stationary solution, the stationary fluid density function and fluid mass function are

$$
\pi_{j}(x)=\lim _{t \rightarrow \infty} \hat{\pi}_{j}(t, x) \quad \text { and } \quad c_{j}(x)=\lim _{t \rightarrow \infty} \hat{c}_{j}(t, x) .
$$

Since we are only considering bounded systems, stability is not an issue. However, the system may have some transient states, and thus the stationary solution may depend on the initial state of the model.

On the continuous intervals of the fluid level distribution, $\pi(x)=\left\{\pi_{j}(x)\right\}$, satisfies [10]

$$
\frac{d}{d x}(\pi(x) \mathbf{R}(x))=\pi(x) \mathbf{Q}(x)
$$

where matrix $\mathbf{Q}(x)$ is the transition rate matrix of the environment process when the fluid level is $x$, and the diagonal matrix $\mathbf{R}(x)=\operatorname{diag}\left\langle r_{j}(x)\right\rangle$ is 
composed by the fluid rates $r_{j}(x), j \in \mathcal{S}^{1}$. The fluid rate determines the rate at which the fluid level changes when the environment is in state $j$ and the fluid level is $x$, i.e., $\frac{d}{d t} X(t)=r_{j}(x)$ when $X(t)=x$ and $M(t)=j$.

To avoid non-ergodic model behaviour we assume that $\mathbf{Q}(x)$ is a bounded irreducible generator matrix, $\mathbf{R}(x)$ is a bounded diagonal matrix for $\forall x \in$ $[0, B]$, such that $\epsilon<\left|r_{j}(x)\right|<\xi$ or $r_{j}(x)=0$, where $\epsilon$ is a small positive number and $\xi$ is the upper bound of the drift ${ }^{2}$ and $Z(t)=\{M(t), X(t)\}$ is irreducible in the sense that there is a valid trajectory from any state $\{i, x\} ; i \in \mathcal{S}, x \in[0, B]$ to any state $\{j, y\} ; j \in \mathcal{S}, y \in[0, B]$ with a positive probability.

In the following, we will use the notation $x^{-}$and $x^{+}$to identify the left and right limit of a function to point $x$ respectively. In particular we will define:

$$
x^{+}=\lim _{h \rightarrow 0^{+}}(x+h), x^{-}=\lim _{h \rightarrow 0^{+}}(x-h) .
$$

The cases when $r_{j}(x)=0$ or when $r_{j}\left(x^{-}\right)$and $r_{j}\left(x^{+}\right)$has different sign result in boundaries of the fluid distribution. The boundary conditions of eq. (1) are discussed in section 4.

Let $0=x_{0}<x_{1}<x_{2}<\ldots<x_{n-1}<x_{n}=B$ denote the set of boundaries, where the fluid distribution might have probability mass. In the first part of this paper we assume that $r_{j}(x) \neq 0, \forall j \in \mathcal{S}$ for $x_{i}<x<x_{i+1}$. The case when $\exists j \in \mathcal{S}$ such that $r_{j}(x)=0$ for $x_{i}<x<x_{i+1}$ is discussed in section 8 .

To obtain the solution of (1) between $x_{i}$ and $x_{i+1}$ we rearrange it to

$$
\frac{d}{d x} \pi(x)=\pi(x)\left(\mathbf{Q}(x)-\frac{d}{d x} \mathbf{R}(x)\right) \mathbf{R}^{-1}(x)=\pi(x) \mathbf{B}(x),
$$

where

$$
\mathbf{B}(x)=\left(\mathbf{Q}(x)-\frac{d}{d x} \mathbf{R}(x)\right) \mathbf{R}^{-1}(x) .
$$

For $x_{i}<x<x_{i+1}$, the solution of (2) can be written as

$$
\pi(x)=\pi\left(x_{i}^{+}\right) \mathbf{W}\left(x_{i}, x\right)
$$

where $\mathbf{W}\left(x_{i}, x_{i}\right)=\mathbf{I}$ and $\mathbf{W}\left(x_{i}, x\right)$ is the solution of

$$
\frac{d}{d x} \mathbf{W}\left(x_{i}, x\right)=\mathbf{W}\left(x_{i}, x\right) \mathbf{B}(x) \text {. }
$$

\footnotetext{
${ }^{1}$ The fact that the generator of the environment process depends on the fluid level prevents us to apply the solution presented, e.g., in [4].

${ }^{2}$ The assumption that $\epsilon<\left|r_{j}(x)\right|<\xi$ or $r_{j}(x)=0$ results that a discontinuity is associated with all sign change.
} 
Various solution methods of eq. (4) are discussed in [5]. In the present paper we neglect the discussion about this kind of inhomogeneous ODEs and refer interested readers to [5].

In the special case when $\mathbf{Q}(x)$ and $\mathbf{R}(x)$ are level independent in the $\left(x_{i}, x_{i+1}\right)$ range, i.e., for $x \in\left(x_{i}, x_{i+1}\right), \mathbf{Q}(x)=\mathbf{Q}_{i}$ and $\mathbf{R}(x)=\mathbf{R}_{i}$ then

$$
\mathbf{B}(x)=\mathbf{Q}_{i} \mathbf{R}_{i}^{-1} \text { and } \mathbf{W}\left(x_{i}, x\right)=e^{\left(x-x_{i}\right) \mathbf{Q}_{i} \mathbf{R}_{i}^{-1}} .
$$

\section{Boundaries and discontinuities}

When at least one element of matrix $\mathbf{R}(x)$ have discontinuity with sign change at level $x_{i}$ we divide the analysis into "pieces" at these $x_{i}$ levels. We assume that the number of pieces are finite. Let $n-1$ the number of possible discontinuities between 0 and $B$ and $x_{1}, x_{2}, \ldots, x_{n-1}$ the points of discontinuities. $x_{0}=0$ is the lower bound and $x_{n}=B$ is the upper bound of the fluid level. Note that, the $\mathbf{R}\left(x_{i}\right)$ values have to be defined at $x_{0}, x_{1}, \ldots, x_{n}$ as well, because they characterize the model behaviour at the discontinuities in the subsequent analytical description.

\subsection{Managing discontinuities}

Discontinuity of matrix $\mathbf{Q}(x)$ do not generate particular problems in the application of the technique. Discontinuity of matrix $\mathbf{R}(x)$ instead can produce probability masses or ambiguous situations if the rate changes sign. In particular, for each discontinuity $x_{i}$ four different situations are possible (See for example [3]). Figure 1 represents those cases. These cases can be further subdivided in other subcases, depending on the value that $\mathbf{R}(x)$ assumes on the discontinuity. In particular, following the terminology given in [3]:

a) and $\mathbf{b})$ : (Emitting states) are states where the sign does not change. Depending on the value of the rate at the discontinuity point, the probability flux simply flows from one side of the discontinuity to the other and no probability mass is formed (if the rate at the discontinuity point has the same sign), or the flow is stopped and some probability mass builds up (if the rate is zero or has the opposite sign). Probability mass coming from other states simply adds to the flux or to the mass depending on the sign of the rate at the discontinuity point. Figure 2a) shows a physical representation of the case where the sign does not change at the environment of $x_{i}$, e.g., $r\left(x_{i}^{-}\right)>0, r\left(x_{i}^{+}\right)>0$ and $r\left(x_{i}\right)>0$. Figure $2 \mathrm{~b}$ ) show the case when the sign of $r\left(x_{i}^{-}\right)$equals with the sign of $r\left(x_{i}^{+}\right)$, but the sign $r\left(x_{i}\right)$ is different, e.g., $r\left(x_{i}^{-}\right)>0, r\left(x_{i}^{+}\right)>0$ and $r\left(x_{i}\right) \leq 0$. In the figures the horizontal move of the ball represents the change of the fluid level at the fluid place. 


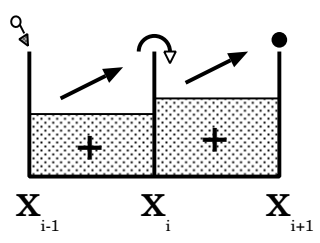

a)

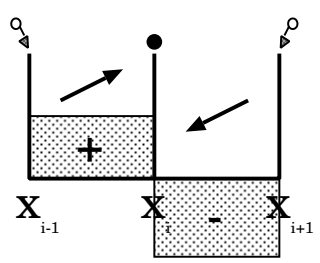

c)

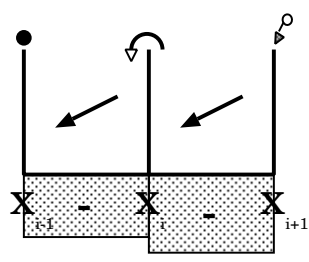

b)

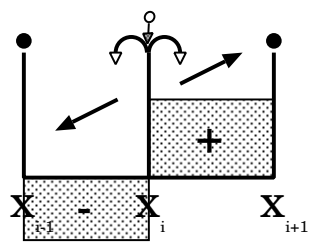

d)

Figure 1: The four possible discontinuity situations

c): (Absorbing states) when the sign changes from positive to negative, the probability accumulates around the discontinuity point, creating thus some probability mass. Probability mass coming from other states, adds to the probability mass generated into this state.

d): (Insulating states) when the sign changes from negative to positive, things becomes a little more complicated. The system is in a situation of unstable equilibrium. No probability mass is generated due to the probability flow. Probability mass coming from other states may either flow to the left, to the right, or stand still forming a probability mass in this state. Placing probability mass in this discontinuity is like placing a ball on the top of a hill (as shown in Figure 3). It may either fall on one side, on the other, or stand still in equilibrium. Which of the three possible behavior will be chosen, depends on the value of the rate at the discontinuity point.

We will now present the equations that in a generic state $j$ couple the probability densities at the beginning $\pi_{j}\left(x_{i}^{+}\right)$and at the end $\pi_{j}\left(x_{i+1}^{-}\right)$of each continuous piece $\left(x_{i}, x_{i+1}\right)$, with the probability masses $c_{j}\left(x_{i}\right)$ in all of the four previous cases.

Case a): positive emitting states $r_{j}\left(x_{i}^{+}\right)>0$ and $r_{j}\left(x_{i}^{-}\right)>0$.

$$
0=c_{j}\left(x_{i}\right) q_{j j}\left(x_{i}\right)+\alpha_{j}^{\leq}\left(x_{i}\right)\left(\pi_{j}\left(x_{i}^{-}\right) r_{j}\left(x_{i}^{-}\right)+\sum_{k \neq j} c_{k}\left(x_{i}\right) q_{k j}\left(x_{i}\right)\right),
$$




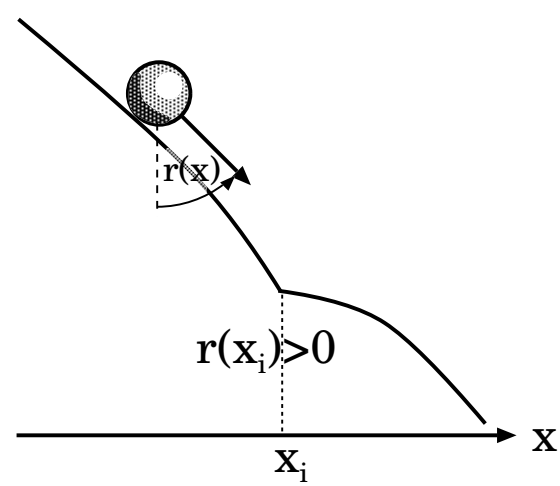

a)

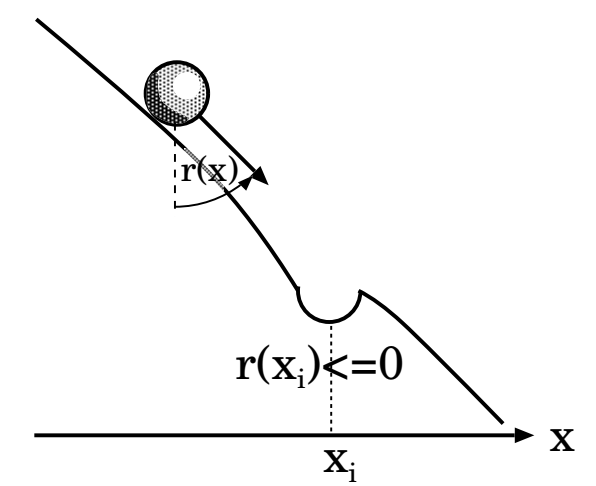

b)

Figure 2: The two possible behavior in the Emitting state

$\pi_{j}\left(x_{i}^{+}\right) r_{j}\left(x_{i}^{+}\right)=\alpha_{j}^{>}\left(x_{i}\right)\left(\pi_{j}\left(x_{i}^{-}\right) r_{j}\left(x_{i}^{-}\right)+\sum_{k \neq j} c_{k}\left(x_{i}\right) q_{k j}\left(x_{i}\right)\right)$,

where $\alpha_{j}^{r e l}\left(x_{i}\right)=\mathbf{1}\left\{r_{j}\left(x_{i}\right)\right.$ "rel" 0$\}$ with $\mathbf{1}\{$.$\} being the indicator function and$ "rel" stands for one of the following relations: $<,=,>, \leq, \geq$. E.g., $\alpha_{j}^{\geq}\left(x_{i}\right)=1$ if $r_{j}\left(x_{i}\right) \geq 0$ and it is 0 otherwise.

The first equation defines the probability mass in state $j$ at fluid level $x_{i}$. It is zero if the probability flows through level $x_{i}$ (i.e. $r_{j}\left(x_{i}\right)>0$ ). If it is not the case the probability mass is equal to the sum of the probability masses coming from neighboring states plus the one that flows from the left piece. The second equation relates the initial probability density of piece $i$ with the final probability density of piece $i-1$. If $r_{j}\left(x_{i}\right)>0$ the difference of the two is coming from the probability masses that other neighboring states may have at fluid level $x_{i}$. If $r_{j}\left(x_{i}\right) \leq 0$ the fluid level is stopped at $x_{i}$ in state $j$, hence $\pi_{j}\left(x_{i}^{+}\right)=0$. These equation can be derived by observing the rate conservation law.

Case b): negative emitting states $r_{j}\left(x_{i}^{+}\right)<0$ and $r_{j}\left(x_{i}^{-}\right)<0$.

$$
\begin{aligned}
0 & =c_{j}\left(x_{i}\right) q_{j j}\left(x_{i}\right)+\alpha_{j}^{\geq}\left(x_{i}\right)\left(-\pi_{j}\left(x_{i}^{+}\right) r_{j}\left(x_{i}^{+}\right)+\sum_{k \neq j} c_{k}\left(x_{i}\right) q_{k j}\left(x_{i}\right)\right), \\
-\pi_{j}\left(x_{i}^{-}\right) r_{j}\left(x_{i}^{-}\right) & =\alpha_{j}^{<}\left(x_{i}\right)\left(-\pi_{j}\left(x_{i}^{+}\right) r_{j}\left(x_{i}^{+}\right)+\sum_{k \neq j} c_{k}\left(x_{i}\right) q_{k j}\left(x_{i}\right)\right) .
\end{aligned}
$$

The equations are identical to the one presented for case a), except for the presence of the minus sign, which is required since the rates are negative. 


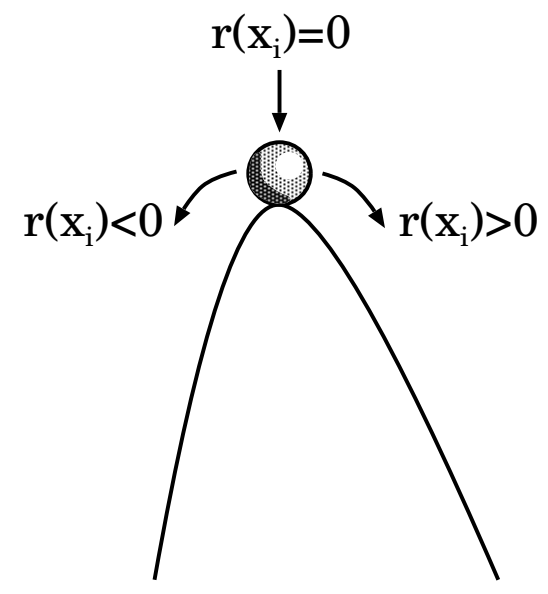

Figure 3: The three possible behavior in the Insulating state

Case c): absorbing states $r_{j}\left(x_{i}^{+}\right)<0$ and $r_{j}\left(x_{i}^{-}\right)>0$.

$$
c_{j}\left(x_{i}\right) q_{j j}\left(x_{i}\right)-\pi_{j}\left(x_{i}^{+}\right) r_{j}\left(x_{i}^{+}\right)+\pi_{j}\left(x_{i}^{-}\right) r_{j}\left(x_{i}^{-}\right)+\sum_{k \neq j} c_{k}\left(x_{i}\right) q_{k j}\left(x_{i}\right)=0 .
$$

In this case we have only one equation that can be used to compute the probability mass $c_{j}\left(x_{i}\right)$ from the probability at the boundaries of the two pieces that are on the two sides of the discontinuity $x_{i}$. Note that we have an expression equal to 0 since $q_{j j}(x)$ is negative by definition.

Case d): isolating states $r_{j}\left(x_{i}^{+}\right)>0$ and $r_{j}\left(x_{i}^{-}\right)<0$.

$$
\begin{aligned}
0 & =c_{j}\left(x_{i}\right) q_{j j}\left(x_{i}\right)+\alpha_{j}^{=}\left(x_{i}\right)\left(\sum_{k \neq j} c_{k}\left(x_{i}\right) q_{k j}\left(x_{i}\right)\right), \\
\pi_{j}\left(x_{i}^{+}\right) r_{j}\left(x_{i}^{+}\right) & =\alpha_{j}^{>}\left(x_{i}\right)\left(\sum_{k \neq j} c_{k}\left(x_{i}\right) q_{k j}\left(x_{i}\right)\right), \\
-\pi_{j}\left(x_{i}^{-}\right) r_{j}\left(x_{i}^{-}\right) & =\alpha_{j}^{<}\left(x_{i}\right)\left(\sum_{k \neq j} c_{k}\left(x_{i}\right) q_{k j}\left(x_{i}\right)\right) .
\end{aligned}
$$

In this case we have three equations: one for the probability mass, and one for the probability density at each side of the boundary. The probability mass coming from the other states is directed along one of those three cases according to the flow rate at the discontinuity point. 


\subsection{Boundary condition}

Boundary condition, at 0 and at the upper boundary $B$ can be seen as special cases of discontinuities. In this case only two different cases per bound arises, depending on the direction of the fluid flow. (In contrast with the cases of discontinuities we use capital letters to denote the cases of boundaries.)

Case A): absorbing states The first case is the one in which the fluid flow is directed towards the bound, that is $r_{j}\left(0^{+}\right)<0$ (for the lower bound), or $r_{j}\left(B^{-}\right)>0$ (for the upper bound). In this case, independently on the sign of the rate at the discontinuity, probability mass builds up at the bound. In this case we can characterize the bound by a single equation:

$$
c_{j}(0) q_{j j}(0)-\pi_{j}\left(0^{+}\right) r_{j}\left(0^{+}\right)+\sum_{k \neq j} c_{k}(0) q_{k j}(0)=0,
$$

for the lower bound, and for the upper bound:

$$
c_{j}(B) q_{j j}(B)+\pi_{j}\left(B^{-}\right) r_{j}\left(B^{-}\right)+\sum_{k \neq j} c_{k}(B) q_{k j}(B)=0 .
$$

Note that the equation is almost identical to the one for the absorbing state in the intermediate discontinuities.

Case B): emitting states The second case is the one in which the fluid flow is directed in the opposite direction with respect to the bound, that is $r_{j}\left(0^{+}\right)>0$ (for the lower bound), or $r_{j}\left(B^{-}\right)<0$ (for the upper bound). In this case we may have two different behaviors depending on the sign of the rate at boundary. No probability mass will be formed if $\operatorname{sign}\left(r_{j}(0)\right)=\operatorname{sign}\left(r_{j}\left(0^{+}\right)\right)$, that is $\alpha_{j}^{>}(0)=1$, and if $\operatorname{sign}\left(r_{j}(B)\right) \operatorname{sign}\left(r_{j}\left(B^{-}\right)\right)$, that is $\alpha_{j}^{<}(B)=1$. Probability mass will instead build up if the sing of the rate at the boundary is zero or opposite to the fluid flow next to the boundary. In any case we will have two equations per boundary, that are:

$$
\begin{aligned}
0 & =c_{j}(0) q_{j j}(0)+\alpha_{j}^{\leq}(0)\left(\sum_{k \neq j} c_{k}(0) q_{k j}(0)\right), \\
\pi_{j}\left(0^{+}\right) r_{j}\left(0^{+}\right) & =\alpha_{j}^{>}(0)\left(\sum_{k \neq j} c_{k}(0) q_{k j}(0)\right)
\end{aligned}
$$

for the lower bound, and for the upper bound:

$$
0=c_{j}(B) q_{j j}(B)+\alpha_{j}^{\geq}(B)\left(\sum_{k \neq j} c_{k}(B) q_{k j}(B)\right),
$$




$$
-\pi_{j}\left(B^{-}\right) r_{j}\left(B^{-}\right)=\alpha_{j}^{<}(B)\left(\sum_{k \neq j} c_{k}(B) q_{k j}(B)\right) .
$$

Note that also in this case the equations are basically identical to the one for the emitting states in the intermediate discontinuities.

\section{The number of equations and unknowns}

If the total number of pieces that composes functions $\mathbf{Q}(x)$ and $\mathbf{R}(x)$ is $n$, and the number of discrete states of the model is $m(m=|\mathcal{S}|)$, then the total number of unknown is $(3 n+1) m$. In particular, each discontinuity has associated a possible probability mass. Since the number of discontinuities is $n+1$, this means that there will be $(n+1) m$ unknowns: one for each discontinuity and state. For each continuous segment, we will be interested in the probability density at the beginning of the segment and the one at the end of the segment ( $2 n$ unknowns). Multiplying this number by the number of states and adding the number of probability masses we obtain $(3 n+1) m$ unknowns.

Theorem 1. The number of equations presented in Section 3 and 4 is equivalent with the number of unknowns, i.e., there are $(3 n+1) m$ equations to determine the $(3 n+1) m$ unknowns.

To prove the theorem we need the following lemma.

Lemma 2. The number of equations (Ne()) given by the arguments of Section 4 for the unknowns associated with state $j$ and the initial and final density values of the first $i(i<n)$ pieces (i.e., $\left.\pi_{j}\left(0^{+}\right), \pi_{j}\left(x_{1}^{-}\right), \pi_{j}\left(x_{1}^{+}\right), \ldots, \pi_{j}\left(x_{i}^{-}\right), \pi_{j}\left(x_{i}^{+}\right)\right)$is $N e(i)=2 i+2$ if the sign of the rate of the last piece is positive $\left(r_{j}\left(x_{i}^{+}\right)>0\right)$, and $N e(i)=2 i+1$ if it is negative $\left(r_{j}\left(x_{i}^{+}\right)<0\right)$.

Proof: By simply counting the equations for each case. For $i=0$ it is true, since for the lower boundary we have one equation $(N e(0)=1)$ if $r_{j}\left(0^{+}\right)<0$ and two equations $(N e(1)=2)$ if $r_{j}\left(0^{+}\right)>0$. By counting the number of equations that are given in each case we have that:

Number of equations per case.

\begin{tabular}{|l|c|c|}
\hline & $r_{j}\left(x_{i-1}^{+}\right)<0$ & $r_{j}\left(x_{i-1}^{+}\right)>0$ \\
\hline$r_{j}\left(x_{i}^{+}\right)<0$ & $2 \quad($ Case $b)$ & $1 \quad($ Case $c)$ \\
$r_{j}\left(x_{i}^{+}\right)>0$ & $3 \quad($ Case $d)$ & $2 \quad($ Case $a)$ \\
\hline
\end{tabular}


Note that since $r_{j}(x)$ has a constant sign over a piece, this implies that $\operatorname{sign}\left(r_{j}\left(x_{i-1}^{+}\right)\right)=\operatorname{sign}\left(r_{j}\left(x_{i}^{-}\right)\right)$. If we express the number of equations $N e(i+1)$ at piece $i+1$ as a function of the number of equations at piece $i$, we have that:

$$
\begin{array}{ll}
N e(i)=N e(i-1)+1=2 i+1=2 i+1 & \text { if } r_{j}\left(x_{i-1}^{+}\right)>0 \text { and } r_{j}\left(x_{i}^{+}\right)<0, \\
N e(i)=N e(i-1)+2=2 i-1+2=2 i+1 & \text { if } r_{j}\left(x_{i-1}^{+}\right)<0 \text { and } r_{j}\left(x_{i}^{+}\right)<0, \\
N e(i)=N e(i-1)+2=2 i+2=2 i+2 & \text { if } r_{j}\left(x_{i-1}^{+}\right)>0 \text { and } r_{j}\left(x_{i}^{+}\right)>0 \\
N e(i)=N e(i-1)+3=2 i-1+3=2 i+2 & \text { if } r_{j}\left(x_{i-1}^{+}\right)<0 \text { and } r_{j}\left(x_{i}^{+}\right)>0 .
\end{array}
$$

Proof of Theorem 1 : There are $n \times m$ equations obtained by the description of the fluid behavior over the continuous pieces (equation (3)), which relates the vectors $\left\{\pi_{j}\left(x_{i-1}^{+}\right)\right\}$with $\left\{\pi_{j}\left(x_{i}^{-}\right)\right\}$.

We still need to show that the boundary conditions presented in section 4 provides $2 n+1$ further equations for every state.

Using Lemma 2 we have that $N e(n-1)=2 n$ if $r_{j}\left(x_{n-1}^{+}\right)>0$ and $N e(n-$ $1)=2 n-1$ if $r_{j}\left(x_{n-1}^{+}\right)<0$. Considering that the number of equations at the upper bound $(B)$ is 1 if $r_{j}\left(x_{n}^{-}\right)>0$ (absorbing upper boundary) and it is 2 if $r_{j}\left(x_{n}^{-}\right)<0$ (emitting upper boundary) the total number of equations associated with state $j$ is $2 n+1$.

\section{Modified system of equations}

Theorem 1 shows that the number of unknowns and the number of equations presented in Section 3 and 4 are equal. Unfortunately, the set of equations presented in Section 3 and 4 has only a trivial solution $\left(c_{j}\left(x_{i}\right)=\pi_{j}\left(x_{i}^{-}\right)=\right.$ $\left.\pi_{j}\left(x_{i}^{+}\right)=0\right)$ in general cases $^{3}$. To overcome this difficulty we introduce a modified system of equations to describe the same fluid system.

We define the probability flux $\varphi_{j}(x)$ and the probability mass flux $d_{j}(x)$ as

$$
\varphi_{j}(x)=\pi_{j}(x) r_{j}(x), \text { and } d_{j}(x)=-c_{j}(x) q_{j j}(x),
$$

and to deal with the probability mass flux we define matrix $A(x)=\left\{a_{j k}(x)\right\}$ as

$$
a_{j k}(x)=\left\{\begin{array}{cl}
\frac{q_{j k}(x)}{-q_{j j}(x)} & \text { if } j \neq k \\
0 & \text { if } j=k
\end{array}\right.
$$

\footnotetext{
${ }^{3}$ Actually, this fact resulted in the major difficulty in our way to find the stationary solution of general fluid systems. This problem is not present in fluid models with single piece, that is why it is not mentioned in previous studies of the subject.
} 


\subsection{Continuous pieces}

Based on equation (1) the probability flux satisfies

$$
\frac{d}{d x} \varphi(x)=\varphi(x)\left(\mathbf{R}^{-1}(x) \mathbf{Q}(x)\right) .
$$

We can use this equation to relate the probability flux at the beginning of piece $i, \varphi\left(x_{i-1}^{+}\right)$, to the probability flux at the end of the same piece, $\varphi\left(x_{i}\right)^{-}$. In particular, we define the flux transition matrix $\mathbf{V}\left(x_{i-1}^{+}, x_{i}^{-}\right)$such that:

$$
\varphi\left(x_{i}^{-}\right)=\varphi\left(x_{i-1}^{+}\right) \mathbf{V}\left(x_{i-1}^{+}, x_{i}^{-}\right) .
$$

By inserting equation (7) into equation (6), we can determine the flux transition matrix by solving the following matrix differential equation up to $x=x_{i}^{-}$:

$$
\frac{d}{d x} \mathbf{V}\left(x_{i-1}^{+}, x\right)=\mathbf{V}\left(x_{i-1}^{+}, x\right)\left(\mathbf{R}^{-1}(x) \mathbf{Q}(x)\right),
$$

with initial condition $\mathbf{V}\left(x_{i-1}^{+}, x_{i-1}^{+}\right)=\mathbf{I}$.

In the special case when $\mathbf{Q}(x)$ and $\mathbf{R}(x)$ are level independent in the $\left(x_{i-1}^{+}, x_{i}^{-}\right)$range, i.e., for $x \in\left(x_{i-1}^{+}, x_{i}^{-}\right), \mathbf{Q}(x)=\mathbf{Q}_{i}$ and $\mathbf{R}(x)=\mathbf{R}_{i}$ then

$$
\mathbf{V}\left(x_{i-1}^{+}, x\right)=e^{\left(x-x_{i-1}\right) \mathbf{R}_{i}^{-1} \mathbf{Q}_{i}} \text {. }
$$

\subsection{Discontinuities}

Case a): positive emitting states $r_{j}\left(x_{i}^{+}\right)>0$ and $r_{j}\left(x_{i}^{-}\right)>0$.

$$
\begin{aligned}
& d_{j}\left(x_{i}\right)=\alpha_{j}^{\leq}\left(x_{i}\right)\left(\varphi_{j}\left(x_{i}^{-}\right)+\sum_{k} d_{k}\left(x_{i}\right) a_{k j}\left(x_{i}\right)\right), \\
& \varphi_{j}\left(x_{i}^{+}\right)=\alpha_{j}^{>}\left(x_{i}\right)\left(\varphi_{j}\left(x_{i}^{-}\right)+\sum_{k} d_{k}\left(x_{i}\right) a_{k j}\left(x_{i}\right)\right) .
\end{aligned}
$$

Case b): negative emitting states $r_{j}\left(x_{i}^{+}\right)<0$ and $r_{j}\left(x_{i}^{-}\right)<0$.

$$
\begin{aligned}
d_{j}\left(x_{i}\right) & =\alpha_{j}^{\geq}\left(x_{i}\right)\left(-\varphi_{j}\left(x_{i}^{+}\right)+\sum_{k} d_{k}\left(x_{i}\right) a_{k j}\left(x_{i}\right)\right), \\
-\varphi_{j}\left(x_{i}^{-}\right) & =\alpha_{j}^{<}\left(x_{i}\right)\left(-\varphi_{j}\left(x_{i}^{+}\right)+\sum_{k} d_{k}\left(x_{i}\right) a_{k j}\left(x_{i}\right)\right) .
\end{aligned}
$$


Case c): absorbing states $r_{j}\left(x_{i}^{+}\right)<0$ and $r_{j}\left(x_{i}^{-}\right)>0$.

$$
d_{j}\left(x_{i}\right)=-\varphi_{j}\left(x_{i}^{+}\right)+\varphi_{j}\left(x_{i}^{-}\right)+\sum_{k} d_{k}\left(x_{i}\right) a_{k j}\left(x_{i}\right) .
$$

Case d): isolating states $r_{j}\left(x_{i}^{+}\right)>0$ and $r_{j}\left(x_{i}^{-}\right)<0$.

$$
\begin{aligned}
d_{j}\left(x_{i}\right) & =\alpha_{j}^{=}\left(x_{i}\right)\left(\sum_{k} d_{k}\left(x_{i}\right) a_{k j}\left(x_{i}\right)\right), \\
\varphi_{j}\left(x_{i}^{+}\right) & =\alpha_{j}^{>}\left(x_{i}\right)\left(\sum_{k} d_{k}\left(x_{i}\right) a_{k j}\left(x_{i}\right)\right), \\
-\varphi_{j}\left(x_{i}^{-}\right) & =\alpha_{j}^{<}\left(x_{i}\right)\left(\sum_{k} d_{k}\left(x_{i}\right) a_{k j}\left(x_{i}\right)\right) .
\end{aligned}
$$

\subsection{Boundary conditions}

Case A): absorbing states $r_{j}\left(0^{+}\right)<0$ (for the lower bound), or $r_{j}\left(B^{-}\right)>0$ (for the upper bound).

$$
\begin{aligned}
& d_{j}(0)=-\varphi_{j}\left(0^{+}\right)+\sum_{k} d_{k}(0) a_{k j}(0), \\
& d_{j}(B)=\varphi_{j}\left(B^{-}\right)+\sum_{k} d_{k}(B) a_{k j}(B) .
\end{aligned}
$$

Case B): emitting states $r_{j}\left(0^{+}\right)>0$ (for the lower bound), or $r_{j}\left(B^{-}\right)<$ 0 (for the upper bound).

$$
\begin{aligned}
d_{j}(0) & =\alpha_{j}^{\leq}(0)\left(\sum_{k} d_{k}(0) a_{k j}(0)\right), \\
\varphi_{j}\left(0^{+}\right) & =\alpha_{j}^{>}(0)\left(\sum_{k} d_{k}(0) a_{k j}(0)\right), \\
d_{j}(B) & =\alpha_{j}^{\geq}(B)\left(\sum_{k} d_{k}(B) a_{k j}(B)\right), \\
-\varphi_{j}\left(B^{-}\right) & =\alpha_{j}^{<}(B)\left(\sum_{k} d_{k}(B) a_{k j}(B)\right) .
\end{aligned}
$$




\subsection{Properties of $\mathbf{W}\left(x_{i}, x\right)$ and $\mathbf{V}\left(x_{i}, x\right)$}

According to the basic rules of the infinitesimal generator of CTMCs the row-sum of $\mathbf{Q}(x)\left(\sum_{k \in \mathcal{S}} Q_{j k}(x)\right)$ must be 0 for $\forall x \geq 0, \forall j \in \mathcal{S}$. One of the main differences between (2) and (6) comes from the fact that $\mathbf{Q}(x)$ is multiplied with $\mathbf{R}^{-1}(x)$ from the right in (2), and from the left in (6). The matrix multiplication of $\mathbf{Q}(x)$ with the $\mathbf{R}^{-1}(x)$ diagonal matrix from the right multiplies the columns of $\mathbf{Q}(x)$ with the associated drift values. This transformation modifies the row-sum. In contrast, the multiplication of $\mathbf{Q}(x)$ with $\mathbf{R}^{-1}(x)$ from the left multiplies the rows of $\mathbf{Q}(x)$ with the associated drift values, hence the row-sum of the product remains 0 for all $x$. We emphasize an important consequence of this property in the following theorem.

Theorem 3. The aggregate probability flux, $\sum_{j \in \mathcal{S}} \varphi_{j}(x)$, remains constant in each continuous intervals, $\left(x_{i-1}^{+}, x_{i}^{-}\right)$, and the row-sum of matrix $\mathbf{V}\left(x_{i-1}^{+}, x_{i}^{-}\right)$ is 1 .

Proof: Let $\mathbb{1}$ be the column vector of ones. The row-sum of matrix $\mathbf{R}^{-1}(x) \mathbf{Q}(x)$ is 0 , hence

$$
\frac{d}{d x} \varphi(x) \mathbb{1}=\varphi(x) \mathbf{R}^{-1}(x) \underbrace{\mathbf{Q}(x) \mathbb{1}}_{0}=0,
$$

i.e., the differential quation (6) preserves the aggregate probability flux in each infinitesimal intervals in $\left(x_{i-1}^{+}, x_{i}^{-}\right)$and so in the whole continuous interval $\left(x_{i-1}^{+}, x_{i}^{-}\right)$.

The theorem on the row-sum of matrix $\mathbf{V}\left(x_{i-1}^{+}, x_{i}^{-}\right)$can be proved by contradiction. Since it has already been proved that the probability flux is constant over a piece, let us consider $\varphi\left(x_{i-1}^{+}\right) \mathbb{1}=\varphi\left(x_{i}^{-}\right) \mathbb{1}=c$. If $\mathbf{V}\left(x_{i-1}^{+}, x_{i}^{-}\right) \mathbb{1} \neq 1$, then we would have:

$$
c=\varphi\left(x_{i}^{-}\right) \mathbb{1}=\varphi\left(x_{i-1}^{+}\right) \mathbf{V}\left(x_{i-1}^{+}, x_{i}^{-}\right) \mathbb{1} \neq \varphi\left(x_{i-1}^{+}\right) \mathbb{1}=c .
$$

\section{Set of equations}

In this section we compose a matrix representation of the set of linear equation describing the behaviour of the fluid system. Basically, we collect the constant coefficients presented in the scalar equations above in a matrix $\mathbf{T}$ such that the row vector of the unknowns $\psi$ is the solution of the equation $\psi \mathbf{T}=0$. We proceed in two steps. First we represent equations (9)-(22) and then equation (7). Figure 4 shows the general structure of the coefficient matrices defined by equations (9)-(22), and of vector $\psi$. 


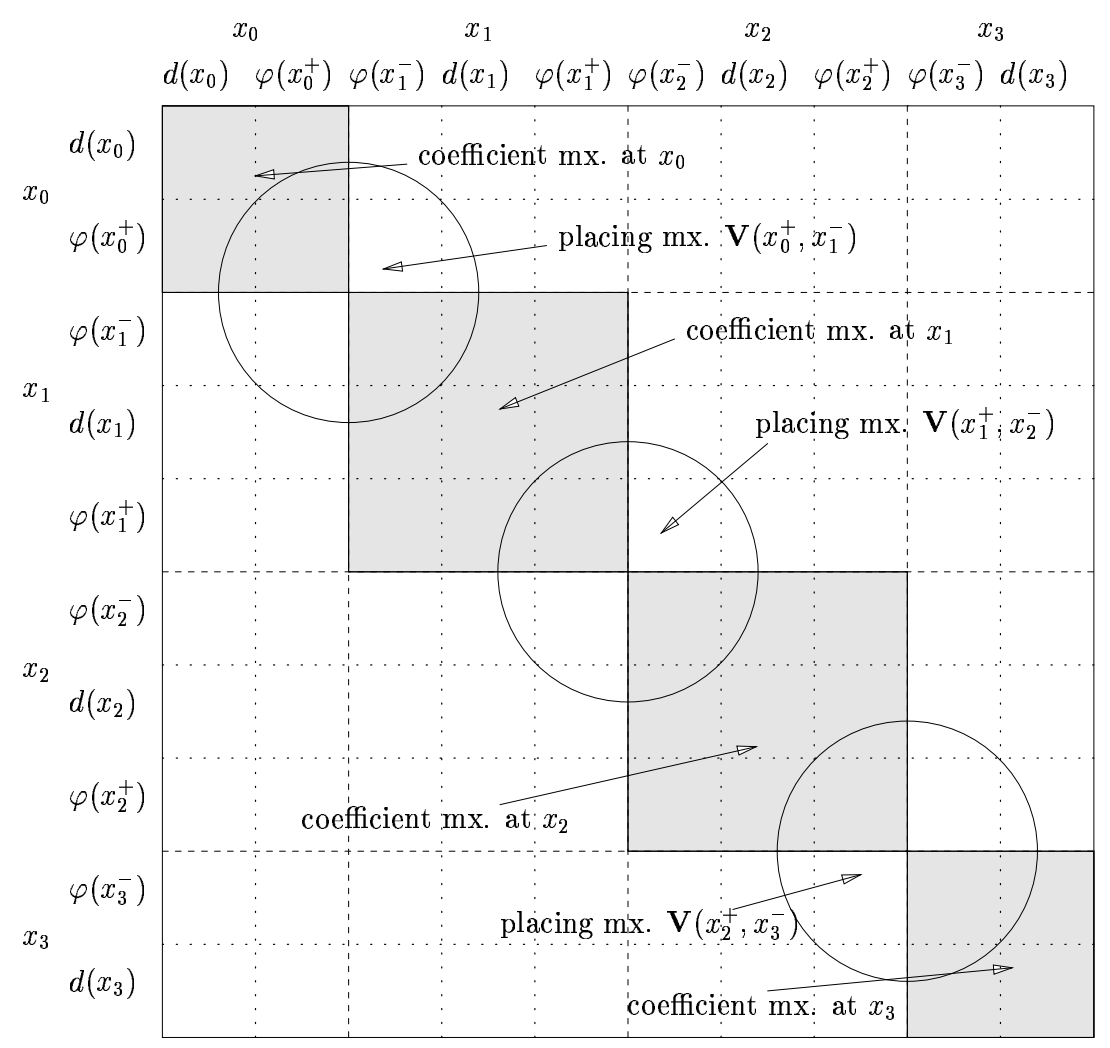

Figure 4: General structure of the coefficient matrix

Considering the restriction that the fluid rate is non-zero between discontinuities we have $2 \times 2 \times 3=12$ different cases with respect to the sign of the fluid rate on the left $\left(r_{j}\left(x_{i}^{-}\right)\right)$, on the right $\left(r_{j}\left(x_{i}^{+}\right)\right)$and at the $i$-th discontinuity $\left(r_{j}\left(x_{i}\right)\right)$. The sign of the rate at the discontinuities $\left(r_{j}\left(x_{i}\right)\right)$ are represented by the $\alpha^{\bullet}\left(x_{i}\right)$ function in the above equations. The different possible signs of the fluid rate on the left $\left(r_{j}\left(x_{i}^{-}\right)\right)$and on the right $\left(r_{j}\left(x_{i}^{+}\right)\right)$of the discontinuity are considered in cases a), b), c) and d). At discontinuity $x_{i}$ we divide the set of states into the following disjoint subsets: $\mathcal{S}_{a}^{(i)}, \mathcal{S}_{b}^{(i)}$, $\mathcal{S}_{c}^{(i)}$ and $\mathcal{S}_{d}^{(i)}$ according to cases a) to d). I.e., $j \in \mathcal{S}_{a}^{(i)}$ if $r_{j}\left(x_{i}^{-}\right)>0$ and $r_{j}\left(x_{i}^{+}\right)>0 ; j \in \mathcal{S}_{b}^{(i)}$ if $r_{j}\left(x_{i}^{-}\right)<0$ and $r_{j}\left(x_{i}^{+}\right)<0 ; j \in \mathcal{S}_{c}^{(i)}$ if $r_{j}\left(x_{i}^{-}\right)>0$ and $r_{j}\left(x_{i}^{+}\right)<0 ; j \in \mathcal{S}_{d}^{(i)}$ if $r_{j}\left(x_{i}^{-}\right)<0$ and $r_{j}\left(x_{i}^{+}\right)>0$. According to this division of states at discontinuity $x_{i}$ matrix $\mathbf{A}\left(x_{i}\right)$ and $\mathbf{V}\left(x_{i-1}^{+}, x_{i}^{-}\right)$are subdivided as follows

$$
\mathbf{A}=\left(\begin{array}{cccc}
\mathbf{A}_{a a} & \mathbf{A}_{a b} & \mathbf{A}_{a c} & \mathbf{A}_{a d} \\
\mathbf{A}_{b a} & \mathbf{A}_{b b} & \mathbf{A}_{b c} & \mathbf{A}_{b d} \\
\mathbf{A}_{c a} & \mathbf{A}_{c b} & \mathbf{A}_{c c} & \mathbf{A}_{c d} \\
\mathbf{A}_{d a} & \mathbf{A}_{d b} & \mathbf{A}_{c d} & \mathbf{A}_{d d}
\end{array}\right), \quad \mathbf{V}=\left(\begin{array}{cccc}
\mathbf{V}_{a a} & \mathbf{V}_{a b} & \mathbf{V}_{a c} & \mathbf{V}_{a d} \\
\mathbf{V}_{b a} & \mathbf{V}_{b b} & \mathbf{V}_{b c} & \mathbf{V}_{b d} \\
\mathbf{V}_{c a} & \mathbf{V}_{c b} & \mathbf{V}_{c c} & \mathbf{V}_{c d} \\
\mathbf{V}_{d a} & \mathbf{V}_{d b} & \mathbf{V}_{c d} & \mathbf{V}_{d d}
\end{array}\right)
$$


where the dependence on the particular discontinuity is neglected for notational simplicity. Using this division of states the structure of the coefficient matrix at discontinuity $x_{i}$ depends on the sign of the rate at the discontinuity $\left(r_{j}\left(x_{i}\right)\right)$ as it is summarized in Figure 5. In the figure, matrix $\mathbf{0}_{u u}$ indicates that equations (9)-(22) do not define the associated quantity and matrix $\mathbf{I}_{u u}$ is the unity matrix of size $\left|\mathcal{S}_{u}\right|$ for $u \in\{a, b, c, d\}$. Matrix $\mathbf{A}_{u u}^{*}$ denotes $\mathbf{A}_{u u}^{*}=-\mathbf{I}_{u u}+\mathbf{A}_{u u}$ (for $\left.u \in\{a, b, c, d\}\right)$.

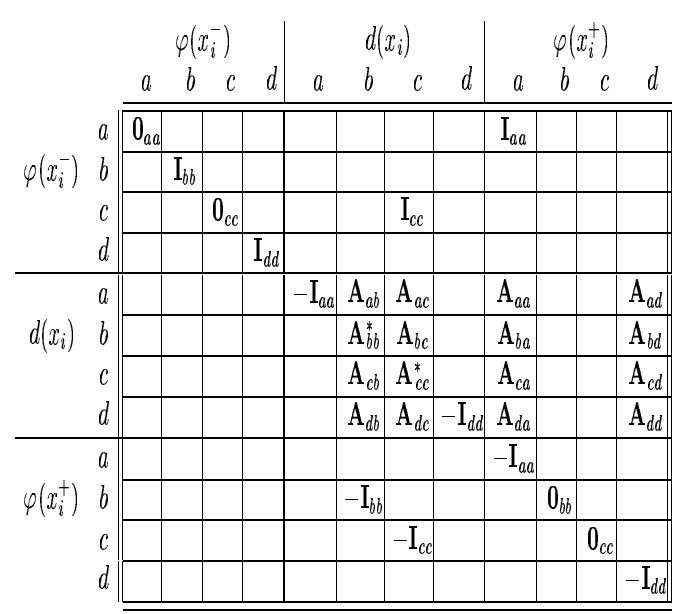

if $r_{j}\left(x_{i}\right)>0$

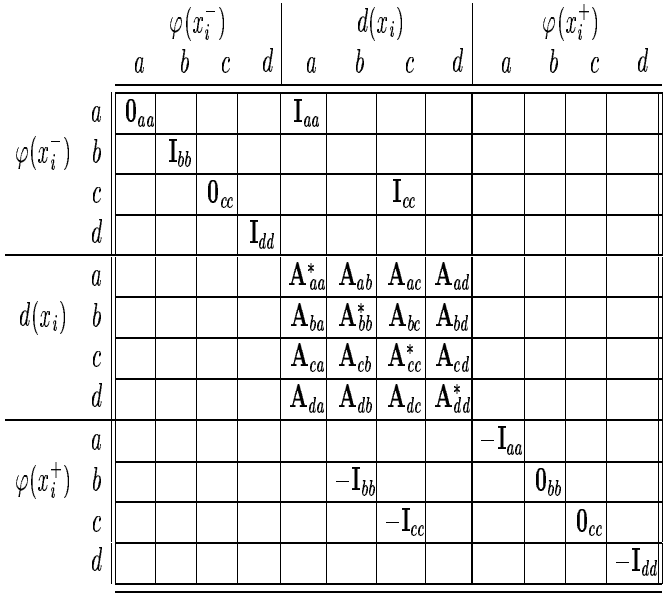

if $r_{j}\left(x_{i}\right)=0$

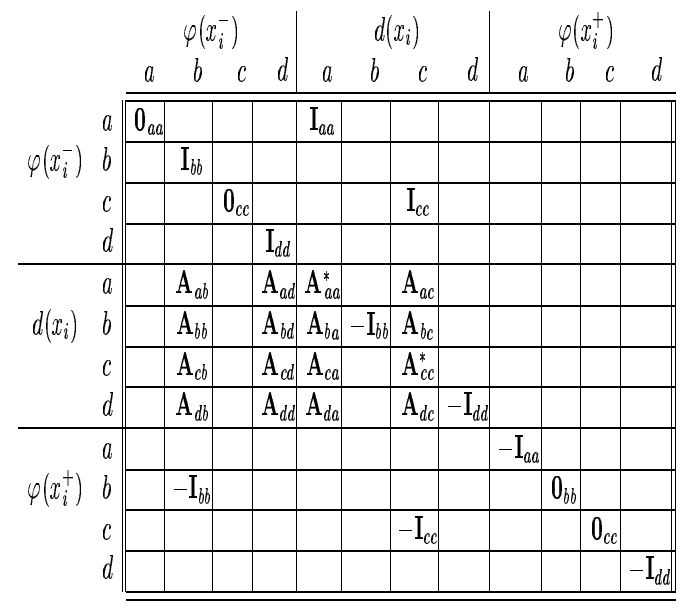

if $r_{j}\left(x_{i}\right)<0$

Figure 5: Coefficient matrix at $x_{i}$

We place the coefficients provided by equation (7) into the idle columns of matrix $\mathbf{T}$, i.e., into the columns of the $\mathbf{0}_{u u}$ matrices The $\mathcal{S}_{a}^{(i)}, \mathcal{S}_{b}^{(i)}, \mathcal{S}_{c}^{(i)}$, $\mathcal{S}_{d}^{(i)}$ partition of $\mathcal{S}$ is associated with discontinuity $x_{i}$. Due to the defini- 
tion of the discontinuities the drift $\left(r_{j}(x)\right)$ can not change sign in the continuous $\left(x_{i-1}^{+}, x_{i}^{-}\right)$interval, hence (as pointed out before) $\operatorname{sign}\left(r_{j}\left(x_{i}^{-}\right)\right)=$ $\operatorname{sign}\left(r_{j}\left(x_{i-1}^{+}\right)\right)$. Based on this property the partitioning at $x_{i-1}\left(\mathcal{S}_{u}^{(i-1)}\right.$, $u \in\{a, b, c, d\})$ is such that

$$
\begin{aligned}
& j \in \mathcal{S}_{a}^{(i)} \Rightarrow j \in \mathcal{S}_{a}^{(i-1)} \bigcup \mathcal{S}_{d}^{(i-1)}, \quad j \in \mathcal{S}_{b}^{(i)} \Rightarrow j \in \mathcal{S}_{b}^{(i-1)} \bigcup \mathcal{S}_{c}^{(i-1)}, \\
& j \in \mathcal{S}_{c}^{(i)} \Rightarrow j \in \mathcal{S}_{a}^{(i-1)} \bigcup \mathcal{S}_{d}^{(i-1)}, \quad j \in \mathcal{S}_{d}^{(i)} \Rightarrow j \in \mathcal{S}_{b}^{(i-1)} \bigcup \mathcal{S}_{c}^{(i-1)}, \\
& \text { i.e., } \mathcal{S}_{a}^{(i)} \bigcup \mathcal{S}_{c}^{(i)}=\mathcal{S}_{a}^{(i-1)} \bigcup \mathcal{S}_{d}^{(i-1)} \text { and } \mathcal{S}_{b}^{(i)} \bigcup \mathcal{S}_{d}^{(i)}=\mathcal{S}_{b}^{(i-1)} \bigcup \mathcal{S}_{c}^{(i-1)} .
\end{aligned}
$$

Figure 6 shows a part of matrix $\mathbf{T}$ with the items obtained from equations (9)-(22) and with the discontinuity dependent partitioning of the states. $u^{\prime}=$ $\mathcal{S}_{u}^{(i-1)}(u \in\{a, b, c, d\})$ denotes the state partitioning according to the sign of the drift around $x_{i-1}$ and matrices $\mathbf{I}_{u v}^{\prime}, \mathbf{V}_{u v}^{\prime}(u, v \in\{a, b, c, d\})$ are obtained from matrices $\mathbf{I}_{u v}, \mathbf{V}_{u v}$ with a reordering of states according to the state partitioning at $x_{i-1}$.

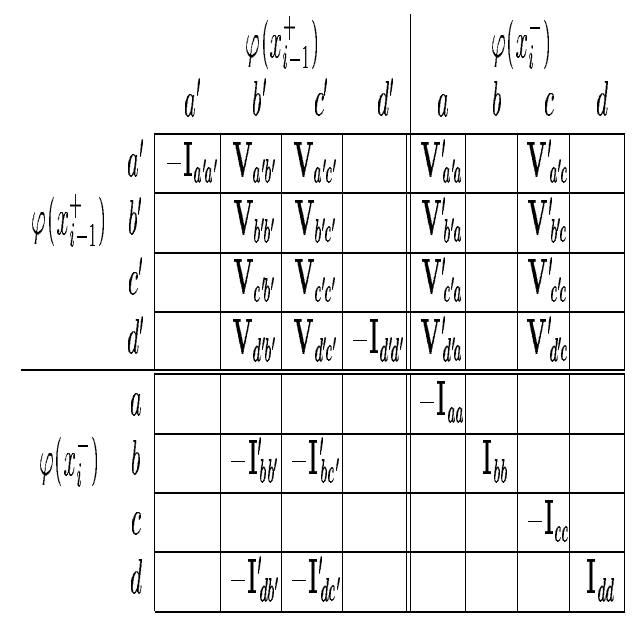

Figure 6: A block of matrix $\mathbf{T}$ with discontinuity dependent state partitioning

Finally, Figure 7 introduces the final structure of the $\mathbf{T}$ matrix at level $x_{i}$. The figure contains all non-zero entries associated with level $x_{i}$. The state partitioning at level $x_{i-1}$ is denoted with $u^{\prime}$ (as before) and at level $x_{i+1}$ is denoted with $u$ " $(u \in\{a, b, c, d\})$. Matrices $\mathbf{I}_{u v}^{\prime}$ and $\mathbf{V}_{u v}^{\prime \prime}$ are defined according to the relevant state partitioning.

The matrix structure in Figure 7 already suggests the following essential property of matrix $\mathbf{T}$.

Theorem 4. The set of equations (9)-(22) and (7) is linearly dependent, and the $\psi \mathbf{T}=0$ equation has non-trivial solution. 


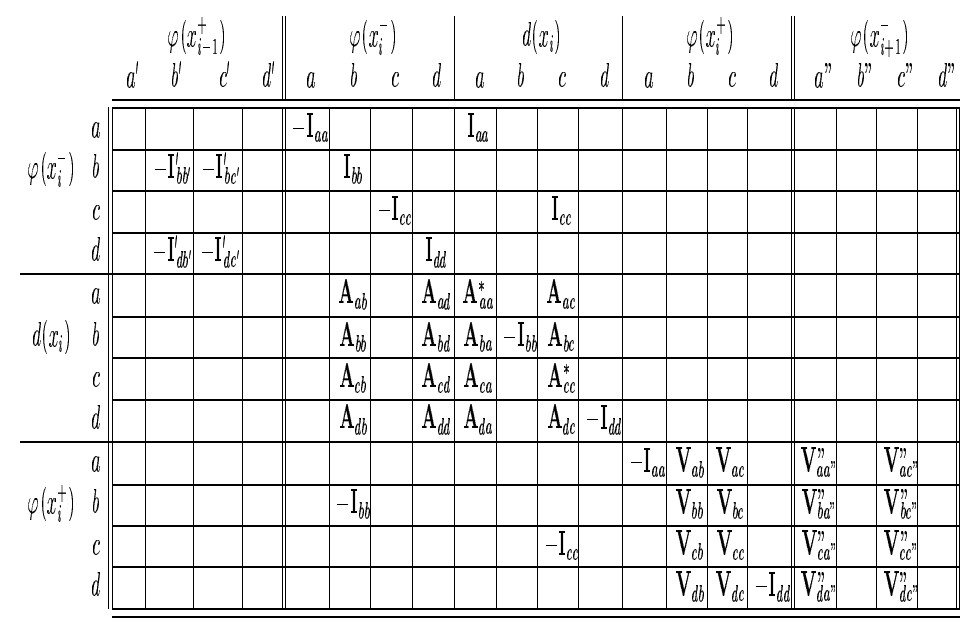

Figure 7: The final structure of the $\mathbf{T}$ matrix at $x_{i}\left(\right.$ with $\left.r_{j}\left(x_{i}\right)<0\right)$

Proof: The row-sum of matrices $\mathbf{A}\left(x_{i}\right)$ and matrices $\mathbf{V}\left(x_{i-1}^{+}, x_{i}^{-}\right)(i \in$ $\{1, \ldots, n\})$ is 1 based on eq. (5) and Theorem 3. By the described construction of matrix $\mathbf{T}$, the row-sum of $\mathbf{T}$ is 0 in all above mentioned possible cases. The theorem is a direct consequence of the row-sum of matrix $\mathbf{T}$.

Note that the introduction of the probability flux $(\varphi(x))$ instead of the fluid density $(\pi(x))$ results in the use of matrix $\mathbf{V}\left(x_{i-1}^{+}, x_{i}^{-}\right)$instead of matrix $\mathbf{W}\left(x_{i-1}^{+}, x_{i}^{-}\right)$, and the introduction of the probability mass flux $(d(x))$ instead of the probability mass $(c(x))$ results in the use of matrix $\mathbf{A}\left(x_{i}\right)$ instead of matrix $\mathbf{Q}\left(x_{i}\right)$. We have to remark however, that Theorem 4, does not ensure that the dimension of the solution space of $\psi \mathbf{T}=0$ is equal to 1 , and thus that $\psi$ can be uniquely determined using some normalizing condition. However, if the system is irreducible and has a unique stationary distribution independent of its initial state, this can be determined using the proposed procedure.

\section{Extension to states with zero rate}

In the previous sections we have always considered $r_{j}(x) \neq 0$ for all the continuous piece. In many practical situation however, there are cases where $r_{j}(x)=0$ for a piece $x_{i}<x<x_{i+1}$. When there are this kind of zero states, both the continuous part and the boundary conditions changes accordingly. 


\subsection{Considering zero states between the discontinu- ities}

Let us denote with $\pi^{0}(x)$ the probability density of the states $j$ where $r_{j}(x)=$ 0 (zero states), and with $\pi^{\emptyset}(x)$ the probability of the other states (non-zero states). We can rewrite equation (1) as:

$$
\begin{aligned}
\frac{d}{d x}\left(\pi^{\emptyset}(x) \mathbf{R}^{\emptyset \emptyset}(x)\right) & =\pi^{\emptyset}(x) \mathbf{Q}^{\emptyset \emptyset}(x)+\pi^{0}(x) \mathbf{Q}^{0 \emptyset}(x) \\
0 & =\pi^{\emptyset}(x) \mathbf{Q}^{\emptyset 0}(x)+\pi^{0}(x) \mathbf{Q}^{00}(x)
\end{aligned}
$$

where $\mathbf{Q}^{\emptyset \emptyset}(x), \mathbf{Q}^{\emptyset 0}(x), \mathbf{Q}^{0 \emptyset}(x), \mathbf{Q}^{00}(x)$ and $\mathbf{R}^{\emptyset \emptyset}(x)$ refers to the sub-matrices of $\mathbf{Q}(x)$ and $\mathbf{R}(x)$ that have elements corresponding to the zero or non-zero states according to their superscript. From equation (23) we obtain:

$$
\begin{aligned}
\pi^{0}(x) & =-\pi^{\emptyset}(x) \mathbf{Q}^{\emptyset 0}(x) \mathbf{Q}^{00^{-1}}(x), \\
\frac{d}{d x}\left(\pi^{\emptyset}(x) \mathbf{R}^{\emptyset \emptyset}(x)\right) & =\pi^{\emptyset}(x)\left[\mathbf{Q}^{\emptyset \emptyset}(x)-\mathbf{Q}^{\emptyset 0}(x) \mathbf{Q}^{00^{-1}}(x) \mathbf{Q}^{0 \emptyset}(x)\right] .
\end{aligned}
$$

Note that

$$
\widehat{\mathbf{Q}}(x)=\mathbf{Q}^{\emptyset \emptyset}(x)-\mathbf{Q}^{\emptyset 0}(x) \mathbf{Q}^{00^{-1}}(x) \mathbf{Q}^{0 \emptyset}(x),
$$

is the generator of the discrete state process restricted to the non-zero states at fluid level $x$.

Let $\widehat{\mathbf{R}}(x)=\mathbf{R}^{\emptyset \emptyset}(x)$ and by changing $\pi(x)$ to $\varphi(x)$ we obtain:

$$
\frac{d}{d x} \varphi^{\emptyset}(x)=\varphi^{\emptyset}(x)\left(\widehat{\mathbf{R}}^{-1}(x) \widehat{\mathbf{Q}}(x)\right) .
$$

The solution of equation (25) can also be expressed in the form

$$
\varphi^{\emptyset}\left(x_{i}^{-}\right)=\varphi^{\emptyset}\left(x_{i-1}^{+}\right) \widehat{\mathbf{V}}\left(x_{i-1}^{+}, x_{i}^{-}\right) .
$$

In the special case when $\mathbf{Q}(x)$ and $\mathbf{R}(x)$ are level independent in the $\left(x_{i-1}, x_{i}\right)$ range, i.e., for $x \in\left(x_{i-1}, x_{i}\right), \mathbf{Q}(x)=\mathbf{Q}_{i}$ and $\mathbf{R}(x)=\mathbf{R}_{i}$ then

$$
\widehat{\mathbf{V}}\left(x_{i-1}, x\right)=e^{\left(x-x_{i-1}\right) \widehat{\mathbf{R}}_{i}^{-1} \widehat{\mathbf{Q}}_{i}} .
$$

We can compute the actual solution of $\pi(x)$ from $\varphi^{\emptyset}(x)$ using the following equations:

$$
\begin{aligned}
\pi^{\emptyset}(x) & =\varphi^{\emptyset}(x) \widehat{\mathbf{R}}^{-1}(x) \\
\pi^{0}(x) & =-\varphi^{\emptyset}(x) \widehat{\mathbf{R}}^{-1}(x) \mathbf{Q}^{\emptyset 0}(x) \mathbf{Q}^{00^{-1}}(x)
\end{aligned}
$$




\subsection{Zero states at the discontinuities}

When one of the pieces around discontinuity $x_{i}$ might have a zero state the set of cases considered in section 6 needs to be extended with the following ones:

\section{Discontinuities}

Case e): $r_{j}\left(x_{i}^{+}\right)=0$ and $r_{j}\left(x_{i}^{-}\right)<0$.

$$
\begin{aligned}
d_{j}\left(x_{i}\right) & =\alpha_{j}^{\geq}\left(x_{i}\right)\left(\sum_{k} d_{k}\left(x_{i}\right) a_{k j}\left(x_{i}\right)\right), \\
\varphi_{j}\left(x_{i}^{+}\right) & =0, \\
-\varphi_{j}\left(x_{i}^{-}\right) & =\alpha_{j}^{<}\left(x_{i}\right)\left(\sum_{k} d_{k}\left(x_{i}\right) a_{k j}\left(x_{i}\right)\right) .
\end{aligned}
$$

Case f): $r_{j}\left(x_{i}^{+}\right)=0$ and $r_{j}\left(x_{i}^{-}\right)>0$.

$$
\begin{aligned}
d_{j}\left(x_{i}\right) & =\varphi_{j}\left(x_{i}^{-}\right)+\sum_{k} d_{k}\left(x_{i}\right) a_{k j}\left(x_{i}\right), \\
\varphi_{j}\left(x_{i}^{+}\right) & =0 .
\end{aligned}
$$

Case g): $r_{j}\left(x_{i}^{+}\right)<0$ and $r_{j}\left(x_{i}^{-}\right)=0$.

$$
\begin{aligned}
d_{j}\left(x_{i}\right) & =-\varphi_{j}\left(x_{i}^{-}\right)+\sum_{k} d_{k}\left(x_{i}\right) a_{k j}\left(x_{i}\right), \\
\varphi_{j}\left(x_{i}^{-}\right) & =0 .
\end{aligned}
$$

Case h): $r_{j}\left(x_{i}^{+}\right)>0$ and $r_{j}\left(x_{i}^{-}\right)=0$.

$$
\begin{aligned}
d_{j}\left(x_{i}\right) & =\alpha_{j}^{\leq}\left(x_{i}\right)\left(\sum_{k} d_{k}\left(x_{i}\right) a_{k j}\left(x_{i}\right)\right), \\
\varphi_{j}\left(x_{i}^{+}\right) & =\alpha_{j}^{>}\left(x_{i}\right)\left(\sum_{k} d_{k}\left(x_{i}\right) a_{k j}\left(x_{i}\right)\right), \\
-\varphi_{j}\left(x_{i}^{-}\right) & =0 .
\end{aligned}
$$

Case i): $r_{j}\left(x_{i}^{+}\right)=0$ and $r_{j}\left(x_{i}^{-}\right)=0$.

$$
\begin{aligned}
d_{j}\left(x_{i}\right) & =\sum_{k} d_{k}\left(x_{i}\right) a_{k j}\left(x_{i}\right), \\
\varphi_{j}\left(x_{i}^{+}\right) & =0 \\
-\varphi_{j}\left(x_{i}^{-}\right) & =0 .
\end{aligned}
$$




\section{Boundary conditions}

Case C): $r_{j}\left(0^{+}\right)=0$.

$$
\begin{aligned}
d_{j}(0) & =\sum_{k} d_{k}(0) a_{k j}(0), \\
\varphi_{j}\left(0^{+}\right) & =0 .
\end{aligned}
$$

Case D): $r_{j}\left(B^{-}\right)=0$.

$$
\begin{aligned}
d_{j}(B) & =\sum_{k} d_{k}(B) a_{k j}(B), \\
\varphi_{j}\left(B^{-}\right) & =0 .
\end{aligned}
$$

These equations can be obtained as special cases of the equations in section 6 by omitting the flux towards the piece with zero state.

\subsection{Number of equations}

Theorem 5. The number of equations presented in this section is equivalent with the number of unknowns, i.e., $(3 n+1) m$.

To prove the theorem we need the following notations and lemma.

Let $m_{i}^{0}$ be the number of zero states in piece $i$. In piece $i$ the matrix equation (26) represents $m-m_{i}^{0}$ equations. We associate each of these equations with a different non-zero state of piece $i$. Let $N e_{j}(i)$ be the number of equations available for determining the unknowns associated with state $j$ at the discontinuities $x_{0}, \ldots, x_{i}$. These unknowns are $d_{j}(0), \varphi_{j}\left(0^{+}\right), \varphi_{j}\left(x_{1}^{-}\right), d_{j}\left(x_{1}\right), \varphi_{j}\left(x_{1}^{+}\right), \ldots, \varphi_{j}\left(x_{i}^{-}\right), d_{j}\left(x_{i}\right), \varphi_{j}\left(x_{i}^{+}\right)$. This way $N e_{j}(i)$ contains the boundary and discontinuity equations associated with pieces $0, \ldots, i$ and one equation of (26) for all pieces on the left of $x_{i}$ where $j$ is a non-zero state.

Lemma 6. For $0 \leq i<d$ the number of equations are $N e_{j}(i)=3 i+2$, if $r_{j}\left(x_{i}^{+}\right)>0, N e_{j}(i)=3 i+2$, if $r_{j}\left(x_{i}^{+}\right)=0$ and $N e_{j}(i)=3 i+1$, if $r_{j}\left(x_{i}^{+}\right)<0$.

\begin{tabular}{|c|c|c|c|}
\hline & $r_{j}\left(x_{i-1}^{+}\right)<0$ & $r_{j}\left(x_{i-1}^{+}\right)=0$ & $r_{j}\left(x_{i-1}^{+}\right)>0$ \\
\hline$r_{j}\left(x_{i}^{+}\right)<0$ & 2 (Case b) & 2 (Case $g)$ & 1 (Case $c)$ \\
\hline$r_{j}\left(x_{i}^{+}\right)=0$ & 3 (Case e) & 3 (Case $i)$ & (Case $f$ ) \\
\hline$r_{j}\left(x_{i}^{+}\right)>0$ & 3 (Case $d)$ & 3 (Case h) & 2 (Case a) \\
\hline
\end{tabular}

Proof of Lemma 6: For $i=0$ the lemma is true. The number of equations at discontinuity $x_{i}$ is:

Number of equations per case. 
Assuming the lemma valid for discontinuity $x_{i-1}$, we have:

$$
\begin{array}{ll}
N e_{j}(i)=N e_{j}(i-1)+2+1=3 i+1 & \text { if } r_{j}\left(x_{i-1}^{+}\right)<0 \text { and } r_{j}\left(x_{i}^{+}\right)<0, \\
N e_{j}(i)=N e_{j}(i-1)+3+1=3 i+2 & \text { if } r_{j}\left(x_{i-1}^{+}\right)<0 \text { and } r_{j}\left(x_{i}^{+}\right)=0, \\
N e_{j}(i)=N e_{j}(i-1)+3+1=3 i+2 & \text { if } r_{j}\left(x_{i-1}^{+}\right)<0 \text { and } r_{j}\left(x_{i}^{+}\right)>0, \\
N e_{j}(i)=N e_{j}(i-1)+2=3 i+1 & \text { if } r_{j}\left(x_{i-1}^{+}\right)=0 \text { and } r_{j}\left(x_{i}^{+}\right)<0, \\
N e_{j}(i)=N e_{j}(i-1)+3=3 i+2 & \text { if } r_{j}\left(x_{i-1}^{+}\right)=0 \text { and } r_{j}\left(x_{i}^{+}\right)=0, \\
N e_{j}(i)=N e_{j}(i-1)+3=3 i+2 & \text { if } r_{j}\left(x_{i-1}^{+}\right)=0 \text { and } r_{j}\left(x_{i}^{+}\right)>0, \\
N e_{j}(i)=N e_{j}(i-1)+1+1=3 i+1 & \text { if } r_{j}\left(x_{i-1}^{+}\right)>0 \text { and } r_{j}\left(x_{i}^{+}\right)<0, \\
N e_{j}(i)=N e_{j}(i-1)+2+1=3 i+2 & \text { if } r_{j}\left(x_{i-1}^{+}\right)>0 \text { and } r_{j}\left(x_{i}^{+}\right)=0, \\
N e_{j}(i)=N e_{j}(i-1)+2+1=3 i+2 & \text { if } r_{j}\left(x_{i-1}^{+}\right)>0 \text { and } r_{j}\left(x_{i}^{+}\right)>0 .
\end{array}
$$

where the number of additive equations describing discontinuity $x_{i}$ and the relation of the flux of non-zero states at the beginning and the end of piece $i$ (equation (26)) are treated separately.

Proof of Theorem 5 : Applying Lemma 6 for the last but one discontinuity $\left(x_{n-1}\right)$ and adding the boundary equations at $B$, we have

$$
\begin{array}{ll}
N e_{j}(n)=N e_{j}(n-1)+2+1=3 n+1 & \text { if } r_{j}\left(x_{n-1}^{+}\right)<0, \\
N e_{j}(n)=N e_{j}(n-1)+2=3 n+1 & \text { if } r_{j}\left(x_{n-1}^{+}\right)=0, \\
N e_{j}(n)=N e_{j}(n-1)+1+1=3 n+1 & \text { if } r_{j}\left(x_{n-1}^{+}\right)>0
\end{array} .
$$

\subsection{Set of linear equations}

Using equation (26) and the discontinuity and boundary equations presented in this section we can compose a similar system of equations as before $(\psi \mathbf{T}=$ 0 ), but the structure of the $\psi$ and the $\mathbf{T}$ matrix is much more complex since we need to introduce 3 subsets of states (with positive, negative and zero drift) which results in 9 cases (a to i) to consider at each discontinuity, in contrast with the 4 cases (a, b, c, d) discussed in Section 7.

Without further explanation we conclude that the main equation set obtained with the presence of zero states remains solvable in a similar way. We only demonstrate the applicability of the approach for the case with zero states via our implementation which automatically generates the $\mathbf{T}$ matrix and solves the linear system also in this case.

\section{Normalizing condition}

The normalizing condition is obtained from the fluid distribution as follows 


$$
\begin{aligned}
1 & =\sum_{j \in \mathcal{S}} \sum_{i=0}^{n} c_{j}\left(x_{i}\right)+\sum_{j \in \mathcal{S}} \sum_{i=1}^{n} \int_{x_{i-1}}^{x_{i}} \pi_{j}(x) d x, \\
& =\sum_{j \in \mathcal{S}} \sum_{i=0}^{n} \frac{d_{j}\left(x_{i}\right)}{-q_{j j}\left(x_{i}\right)}+\sum_{j \in \mathcal{S}} \sum_{i=1}^{n} \int_{x_{i-1}}^{x_{i}} \frac{\varphi_{j}(x)}{r_{j}(x)} d x .
\end{aligned}
$$

Unfortunately, the normalizing condition can not be evaluated based on the $\mathbf{V}\left(x_{i-1}, x_{i}\right)$ matrices, but it also requires the evaluation of the $\int_{x_{i-1}}^{x_{i}} \pi_{j}(x) d x$ integral.

When some of the states have associated zero drift in a continuous piece (6) is no longer valid and the integrals of the probability densities are calculated based on (24) instead of (1).

The normalizing condition can also be expressed using a normalizing vector $\eta$. In this case the normalizing condition becomes: $\psi \eta^{T}=1$.

Vector $\eta$ can be constructed from the definition of $\psi$ and equations (45) and (46). In particular, from (26) and (27) we may compute for each pieces $\left(x_{i-1}, x_{i}\right)$ :

$$
\begin{aligned}
\int_{x_{i-1}}^{x_{i}} \pi^{\emptyset}(x) d x & =\varphi^{\emptyset}\left(x_{i-1}^{+}\right) \int_{x_{i-1}}^{x_{i}} \widehat{\mathbf{V}}\left(x_{i-1}^{+}, x\right) \widehat{\mathbf{R}}^{-1}(x) d x \\
\int_{x_{i-1}}^{x_{i}} \pi^{0}(x) d x & =-\varphi^{\emptyset}\left(x_{i-1}^{+}\right) \int_{x_{i-1}}^{x_{i}} \widehat{\mathbf{V}}\left(x_{i-1}^{+}, x\right) \widehat{\mathbf{R}}^{-1}(x) \mathbf{Q}^{\emptyset 0}(x) \mathbf{Q}^{00^{-1}}(x) d x .
\end{aligned}
$$

Introducing

$$
\begin{aligned}
U_{i}^{\emptyset} & =\int_{x_{i-1}}^{x_{i}} \widehat{\mathbf{V}}\left(x_{i-1}^{+}, x\right) \widehat{\mathbf{R}}^{-1}(x) d x \\
U_{i}^{0} & =-\int_{x_{i-1}}^{x_{i}} \widehat{\mathbf{V}}\left(x_{i-1}^{+}, x\right) \widehat{\mathbf{R}}^{-1}(x) \mathbf{Q}^{\emptyset 0}(x) \mathbf{Q}^{00^{-1}}(x) d x,
\end{aligned}
$$

we have

$$
\begin{aligned}
\sum_{j \in \mathcal{S}} \int_{x_{i-1}}^{x_{i}} \pi_{j}(x) d x & =\int_{x_{i-1}}^{x_{i}} \pi^{\emptyset}(x) d x \mathbb{1}+\int_{x_{i-1}}^{x_{i}} \pi^{0}(x) d x \mathbb{1}= \\
& =\varphi^{\emptyset}\left(x_{i-1}^{+}\right)\left[U_{i}^{\emptyset} \mathbb{1}+U_{i}^{0} \mathbb{1}\right] .
\end{aligned}
$$

Using the row vectors $\eta_{i}^{(\varphi)}=\left(U_{i}^{\emptyset} \mathbb{1}+U_{i}^{0} \mathbb{1}\right)^{T}$, and $\eta_{i}^{(d)}=\left\{\frac{1}{-q_{j j}\left(x_{i}\right)}\right\}, j \in \mathcal{S}$, we can build the normalizing vector $\eta$ as follows:

$$
\eta=[\underbrace{\eta_{0}^{(d)}}_{d(0)}, \underbrace{\eta_{1}^{(\varphi)}, \underline{0}_{1}^{0}}_{\varphi\left(0^{+}\right)}, \underbrace{\underline{0}}_{\varphi\left(x_{1}^{-}\right)}, \underbrace{\eta_{1}^{(d)}}_{d\left(x_{1}\right)}, \underbrace{\eta_{2}^{(\varphi)}, \underline{0}_{2}^{0}}_{\varphi\left(x_{1}^{+}\right)}, \underbrace{\underline{0}}_{\varphi\left(x_{2}^{-}\right)}, \ldots, \underbrace{\eta_{n}^{(d)}}_{d(B)}],
$$


where $\underline{0}_{i}^{0}$ and $\underline{0}$ are the row vectors with as many zero components as the number of zero states in piece $i$, and the number of states, respectively. The under braces indicate the $\varphi$ vector elements associated with the particular $\eta$ vector elements. Note that the non-zero $\eta$ elements are associated with the discontinuities $\left(\eta_{i}^{(d)}\right)$ and the states of non-zero rate at the lower boundary of continuous pieces $\left(\eta_{i}^{(\varphi)}\right)$.

\section{Numerical example}

To demonstrate the solution method introduced in the previous sections we evaluate various versions of the high-speed network model presented in [4].

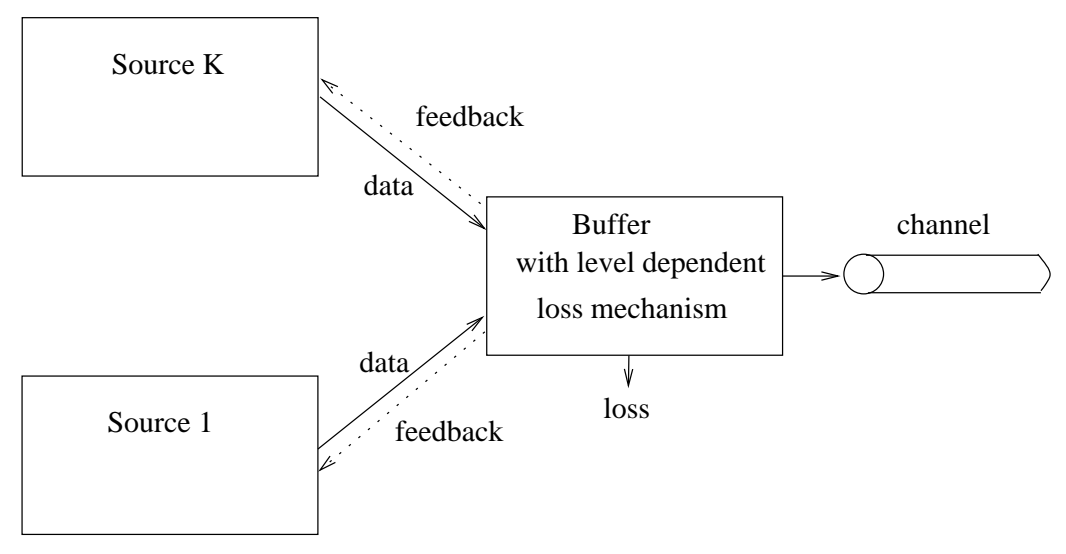

Figure 8: The multiplexer model

The model is composed by $K$ identical 2 state sources and a buffer (see Figure 8). The sources generate data with $J(1, \ldots, J)$ different priorities. The traffic behaviour of each source is governed by a 2-state Markov chain with generator $\mathbf{G}(x)=\left[\begin{array}{cc}-\alpha(x) & \alpha(x) \\ \beta(x) & -\beta(x)\end{array}\right]$, where $x$, the fluid level, represents the queue length in the buffer. When the Markov chain of a source is in state $i$ at queue length $x$ the source generates priority $j$ data at rate $\lambda_{i}^{(j)}(x)$. To reduce the probability of buffer overflow the buffer drops lower priority data, when the buffer level is high. The $B_{1}, B_{2}, \ldots, B_{J-1}$ levels determine the dropping mechanism. When the buffer content is higher than $B_{j}$ the buffer drops all incoming priority $j$ data. In state $i$ and fluid level $x$ the actual rate of a source is $\lambda_{i}(x)=\sum_{j: x>B_{j}} \lambda_{i}^{(j)}(x)$.

Case I: When the source behaviour $\left(\mathbf{G}(x)\right.$ and $\left.\lambda_{i}^{(j)}(x)\right)$ is independent of 
the buffer level, i.e., $\mathbf{G}(x)=\left[\begin{array}{cc}-\alpha & \alpha \\ \beta & -\beta\end{array}\right]$ and $\lambda_{i}^{(j)}(x)=\lambda_{i}^{(j)}$, the obtained model is identical with the one in [4], hence we can compare the results obtained there with the results of our solution method.

The basic set of model parameters is the following:

\section{Model parameters}

\begin{tabular}{|c|c|l|}
\hline$K$ & 20 & number of sources \\
$\alpha$ & 0.4 & transition rate $(\mathrm{OFF} \rightarrow \mathrm{ON})$ \\
$\beta$ & 1.0 & transition rate $(\mathrm{ON} \rightarrow \mathrm{OFF})$ \\
$C$ & 11.428 & channel capacity \\
$J$ & 2 & number of priority levels \\
$\lambda_{1}^{(1)}=\lambda_{1}^{(2)}$ & 0 & data generation in OFF state \\
$\lambda_{2}^{(1)}$ & 1.0 & low priority data generation \\
$\lambda_{2}^{(2)}$ & 0.5 & high priority data generation \\
$B$ & 1.5 & buffer size \\
$B_{1}$ & $0.4-1.2$ & threshold of dropping low priority data \\
$B_{2}$ & $B$ & threshold of dropping high priority data \\
\hline
\end{tabular}

In the following cases we indicate only the modified values with respect to this basic set of parameters.

Figure 9 depicts the joint distribution of the buffer content and the system state when the parameters are taken from the basic parameter set.

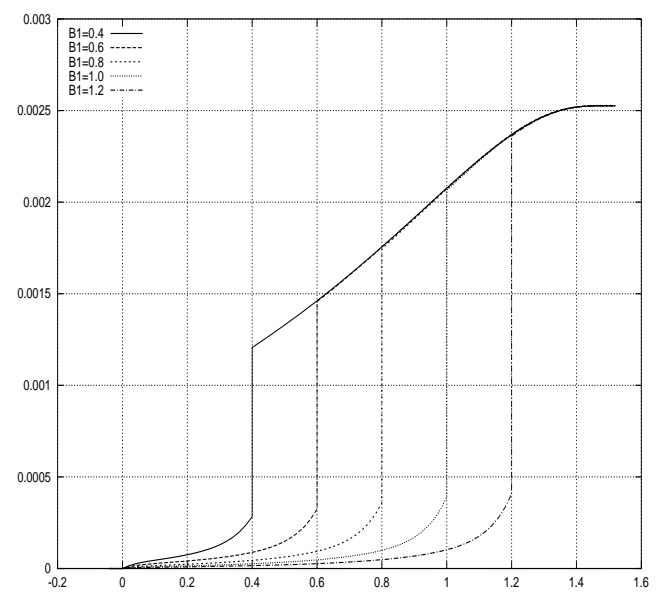

a) 8 active users

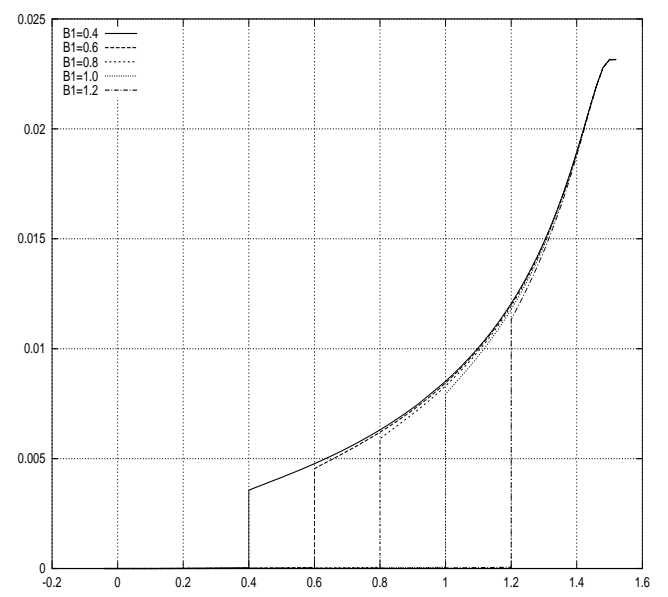

b) 10 active users

Figure 9: Buffer content distribution with 8 and 10 active users (Case I)

To compare the results with the one presented in [4], we also evaluate the loss probability of the low priority data. Since the exact loss probability 
values are not given in [4] we only present figure 10, which is supposed to be identical with figure $4 \mathrm{a}$ of [4] and leave the verification for the reader.

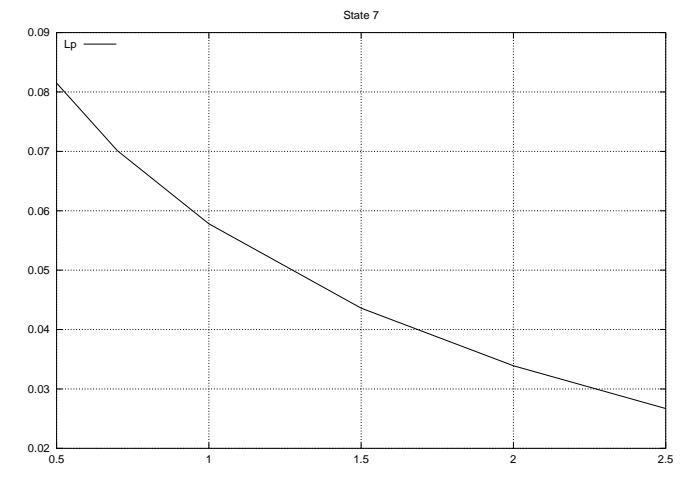

Figure 10: Loss probability of priority 1 data

Case II: Increasing the number of sources to $K=30$, changing $B=1$, $C=17.142$ and varying $B_{1}$ from 0.3 to 0.7 results the distribution presented in Figure 11.

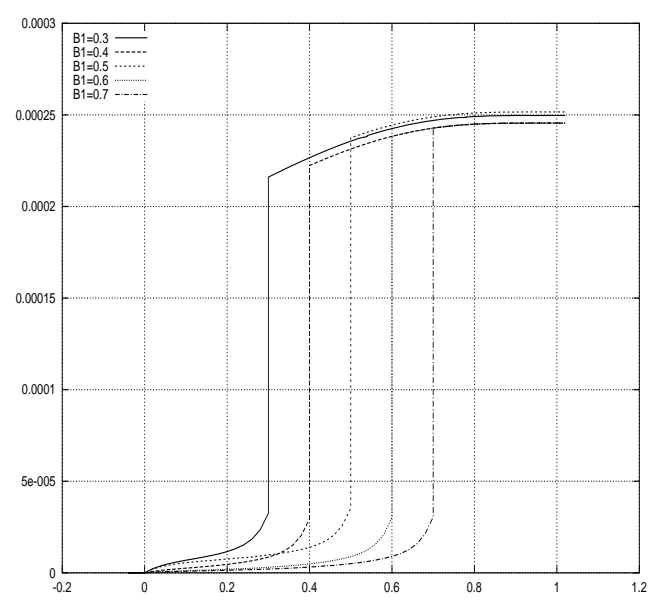

a) 12 active users

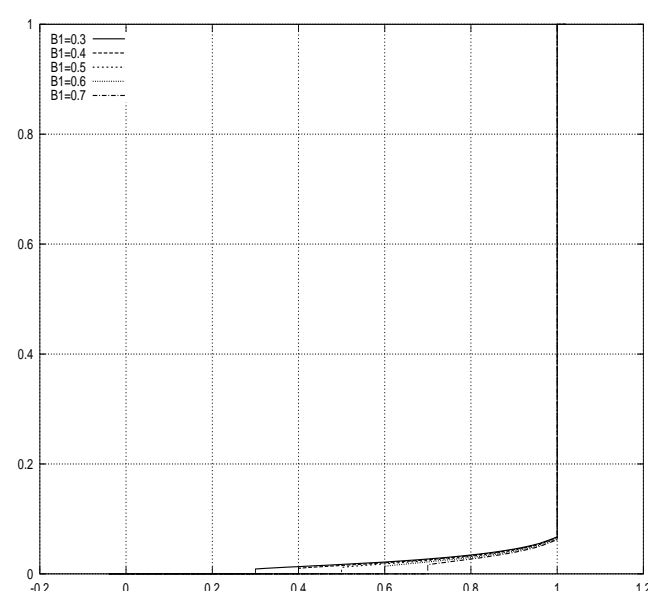

b) overall distribution

Figure 11: Buffer content distribution with 30 sources (Case II)

Case I and II can also be evaluated with the method presented in [4]. The next case instead can no longer be solved using the approach proposed in [4].

Case III: In the following we assume a more sophisticated system behaviour. The sources adapt their behaviour to the actual dropping of the buffer, which becomes known for the sources via feedback signals (e.g., missing acknowledgements). There are two ways to adjust the source behaviour. 
The source might change the data generation rate and also the governing Markov chain. The procedure presented in the paper allows to consider both of these adjustments. First we make the governing Markov chain buffer level dependent such that $\alpha(x)=\left\{\begin{array}{ll}1.0 & \text { if } x<B_{1} \\ a_{2} & \text { if } B_{1}<x<B\end{array}\right.$, where $a_{2}$ varies between 0.001 and 1 . The rest of the modified model parameters with respect to the basic set are $B=1, B_{1}=0.7$. Figure 12 provides the buffer content distribution in this case.

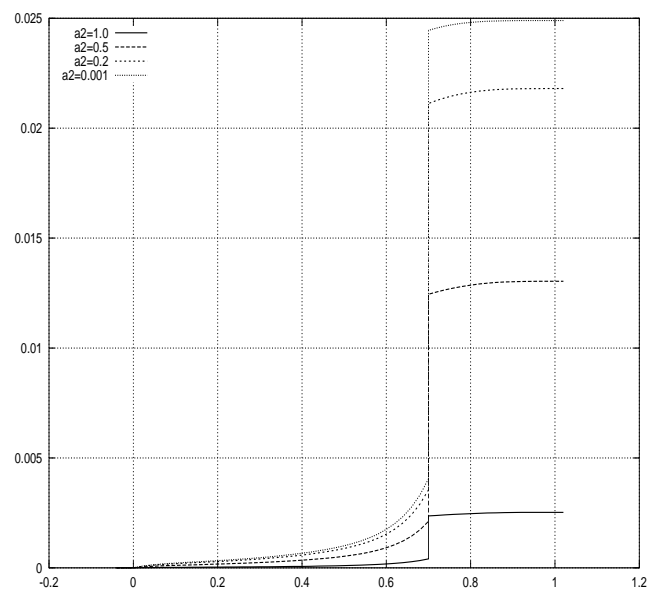

a) 8 active users

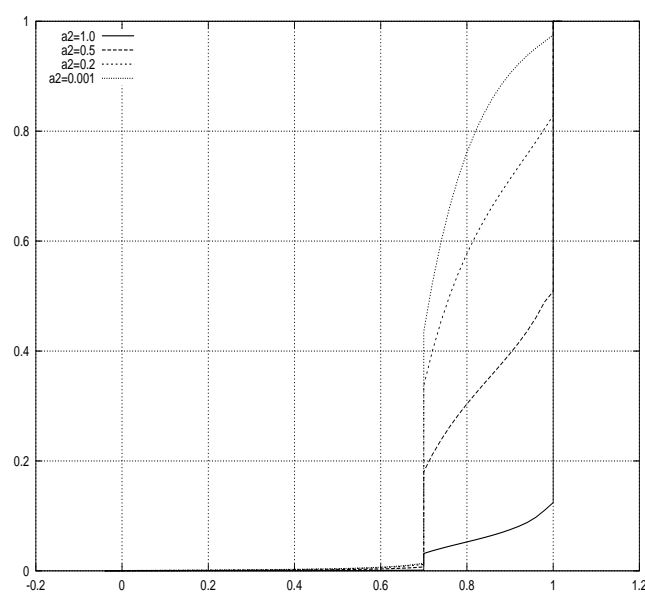

b) overall distribution

Figure 12: Buffer content distribution with adaptive source behaviour (Case III.)

Case IV: Finally, we consider a model behaviour which contains isolating state as well. (Isolating state is not allowed in [4].) In this version of the model the data accumulation rate depends on the buffer level, such that the channel capacity drops down when the buffer is heavily loaded, $C(x)=\left\{\begin{array}{cl}11.428 & \text { if } x<B_{1} \\ s 2 \cdot 11.428 & \text { if } B_{1}<x<B\end{array}\right.$, where $s 2$ varies between $10 \%$ and $100 \%$. This may happen, for example, when the system drops packets at high buffer level. Similar to Case III, $B=1$ and $B_{1}=0.7$. The distributions are given in Figure 13. Figure 13a reflects the expectations based on the physical understanding of the model. The lower is the system capacity at high buffer levels the higher is the buffer content. (Curves closer to the horizontal axes means higher average buffer level.)

The proposed numerical procedure is composed by 3 main sets:

- calculation of $\mathbf{V}\left(x_{i}, x_{i+1}\right)$ matrices (eq. (8)),

- solving the linear system of equations $\varphi \mathbf{T}=0$, 


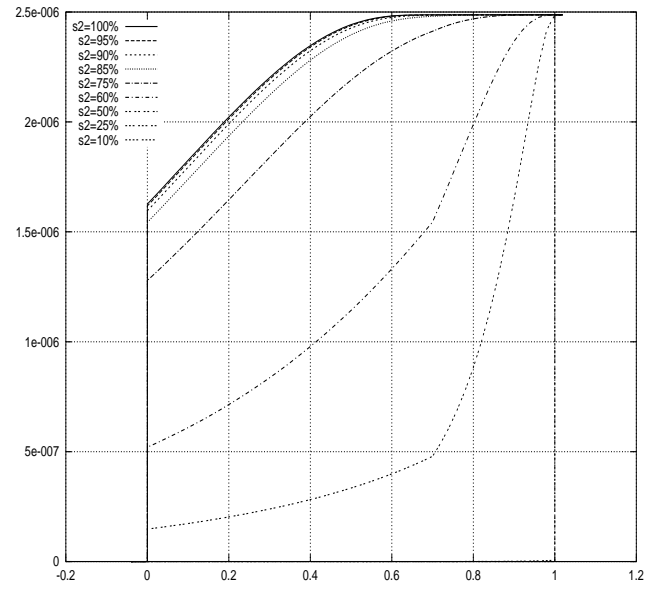

a) 4 active users

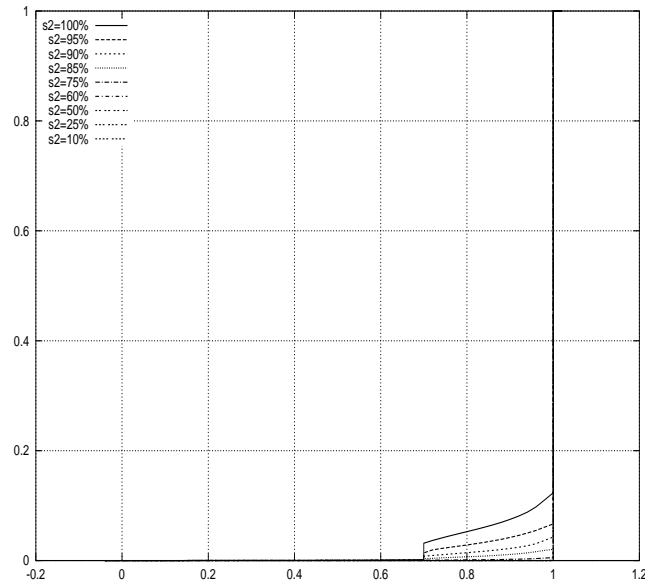

b) overall distribution

Figure 13: Buffer content distribution with varying channel capacity (Case IV.)

- normalizing the solution (eq. (46)).

The computational complexity of these steps are characterized by the following model parameters. The cardinality of matrix $\mathbf{V}\left(x_{i}, x_{i+1}\right)$ is $m \times m$, while the cardinality of matrix $\mathbf{T}$ is $m(3 n+1) \times m(3 n+1)$. Matrices $\mathbf{V}\left(x_{i}, x_{i+1}\right), 0 \leq i<n$ are complete matrices and should be stored in complete matrix form, while matrix $\mathbf{T}$ has a regular block structure which can be exploited to reduce memory usage and speed up computation. Equation (8) is solved $n$ times. The possible computational methods for solving (8) are detailed in [6] and [5] together with their computational complexity. We applied Gauss elimination for solving the linear system. For all presented examples, the computation of the $d(x)$ and $\varphi(x)$ vectors took less than a minute with our $\mathrm{C}$ implementation on regular $\mathrm{PC}$.

According to our experiences the main limitation of the proposed method is not the complexity of the computation, but its numerical stability. Depending on the model parameters the elements of the $\mathbf{V}\left(x_{i}, x_{i+1}\right)$ matrices might become extremely large (as it is discussed in [5]) and the linear system becomes ill conditioned. In some cases, the division of continuous pieces by the introduction of "artificial" discontinuity points helps to improve the numerical properties (because it decreases the large values in the $\mathbf{V}\left(x_{i}, x_{i+1}\right)$ matrices associated with the divided continuous pieces). We observed that the elements of the $\mathbf{V}\left(x_{i}, x_{i+1}\right)$ matrices should be less than $10^{15}$ for all continuous pieces to obtain a reasonable solution with our implementation. 


\section{Conclusion}

The stationary solution of bounded fluid models with fluid level dependent drift and transition matrix is considered in this paper. The set of equation to characterize the unknown quantities of the stationary distribution has a non-linear dependence on the applied system measures.

The commonly applied system measures, the fluid density and the fluid mass functions, result in over-determined system of equations in case of more than one continuous pieces. Due to the non-linear dependence of the equation system on the applied system measures we could prove that a given linear transformation of the system measures results in a solvable set of equations.

We also implemented the computational method based on the transformed system measures and provided numerical examples using the proposed analysis method.

\section{References}

[1] D. Anick, D. Mitra, and M. M. Sondhi. Stochastic theory of a datahandling system. Bell System Thechnical Journal, 61(8):1871-1894, Oct. 1982 .

[2] S. Asmussen. Stationary distributions for fluid flow models with or without brownian noise. Stochastic Models, 11(4):21-49, 1995.

[3] D.-Y. Chen, Y. Hong, and K. S. Trivedi. Second order stochastic fluid flow models with fluid dependent flow rates. Performance Evaluation, 49(1-4):341-358, 2002.

[4] A. I. Elwalid and D. Mitra. Statistical multiplexing with loss priorities in rate-based congestion control of high-speed networks. IEEE Transaction on communications, 42(11):2989-3002, Nov. 1994.

[5] R. German, M. Gribaudo, G. Horváth, and M. Telek. Stationary analysis of FSPNs with mutually dependent discrete and continuous parts. In International Conference on Petri Net Performance Models - PNPM 2003, pages 30-39, Urbana, IL, USA, Sep. 2003. IEEE CS Press.

[6] M. Gribaudo and R. German. Numerical solution of bounded fluid models using matrix exponentiation. In Proc. 11th GI/ITG Conference on Measuring, Modelling and Evaluation of Computer and Communication Systems (MMB), Aachen, Germany, Sep. 2001. VDE Verlag. 
[7] G. Horton, V. G. Kulkarni, D. M. Nicol, and K. S. Trivedi. Fluid stochastic Petri nets: theory, application, and solution techniques. European Journal of Operations Research, 105(1):184-201, Feb. 1998.

[8] O. Kella and W. Stadje. Exact results for a fluid model with statedependent flow rates. Probability in the Engineering and Informational Sciences, 16(4):389-402, 2002.

[9] D. P. Kroese and Scheinhardt W. R. W. Joind distributions for interacting fluid queues. Queueing Systems, 37:99-139, 2001.

[10] V. G. Kulkarni. Fluid models for single buffer systems. In J. H. Dshalalow, editor, Models and Applications in Science and Engineering, Frontiers in Queueing, pages 321-338. CRC Press, 1997.

[11] L. C. G. Rogers. Fluid models in queueing theory and Wiener-Hopf factorization of Markov chains. Annals of Applied Probability, 4(2):390413, 1994.

[12] K. Wolter. Second order fluid stochastic petri nets: an extension of GSPNs for approximate and continuous modelling. In Proc. of World Congress on System Simulation, pages 328-332, Singapore, Sep. 1997. 